COO-3285-8

May 1973

\title{
Broken Conformal Symmetry and Hadronic Scaling $\$ *$
}

\author{
B. C. Yunn \\ Department of Physics, The Johns Hopkins University \\ Baltimore, Maryland 21218
}

NOTICE

This report was prepared as an account of work sponsored by the United States Government. Neither the United States nor the United States Atomic Energy Commission, nor any of their employees, nor any of Commission, nor any of their employees, nor any of their contractors, subcontractors, or their employees, makes any warranty, express or implied, or assumes any legal liability or responsibility for the accuracy, com-
pleteness or usefulness of any information, apparatus, pleteness or usefulness of any information, ap paratus, would not infringe privately owned rights.

1

Technical Report 


\section{DISCLAIMER}

This report was prepared as an account of work sponsored by an agency of the United States Government. Neither the United States Government nor any agency Thereof, nor any of their employees, makes any warranty, express or implied, or assumes any legal liability or responsibility for the accuracy, completeness, or usefulness of any information, apparatus, product, or process disclosed, or represents that its use would not infringe privately owned rights. Reference herein to any specific commercial product, process, or service by trade name, trademark, manufacturer, or otherwise does not necessarily constitute or imply its endorsement, recommendation, or favoring by the United States Government or any agency thereof. The views and opinions of authors expressed herein do not necessarily state or reflect those of the United States Government or any agency thereof. 


\section{DISCLAIMER}

Portions of this document may be illegible in electronic image products. Images are produced from the best available original document. 
Abstract

High energy, large momentum transfer hadronic reactions are studied in the framework of a broken conformal symmetry. In the conformal symmetry limit, hadrons are assigned phenomenological fields. which belong to infinite dimensional irreducible representations of a pseudo unitary group SU $(2,2)$, which is homomorphic to the conformal group, and also their effective action is conformal invariant. Studying simple Lagrangian models, we conjecture that the conformal symmetry is spontaneously broken and also that the scalar field $\chi$ (the Goldstone boson), which has nonvanishing vacuum expectation value, has a scale dimension $d=-2+2 \delta$ where $\delta$ represents deviation from the canonical dimension. We then calculate the inverse propagator up to and including the first order in the tadpole expansion explicitly and find that hadrons lie on almost linear Regge trajectories, which are determined by regular null surfaces of the inverse propagator. The widths of resonances are connected to the anomalous part of the dimension, $\mathcal{S}$. Empirical fit with the data (baryon resonances) gives $\delta \sim 0.04$. In this framework we also study inclusive reactions of the type $p_{1}+p_{2} \rightarrow q+X$ in regions of high transverse momenta. On studying the absorptive part of the six-point conformal amplitude, we find that the single particle distributions exhibit a parton-like structure and also obey simple sealing law6; presently available experimental data on the reaction $\mathrm{pp} \rightarrow \pi^{\circ} \mathrm{X}$ support this conclusion. We also predict large rates of heavy particles $(K, \boldsymbol{\eta})$ production. 
Abstract

Page

Table of Contents

Chapter 1 Introduction a l

Chapter 2 On Conformal Invariant Theories 10

2.1) Conformal Group 10

2. 2) Induced Representations of $\operatorname{SU}(2,2) \quad 13$

2.3) Anomalous Dimension 18

2.4) Effective Action 21

2. 5) Construction of Conformal Amplitudes; 27 Diagram Rules

2.6) Some Remariks on $\mathrm{SU}(2,2)$ Invariant 32 Amplitudes

2. 7) Problems with Fermions 35

Chapter 3 Broken Conformal Symmetry 38

3. 1) Formulation of the Problem 38

3. 2) Inverse Propagator; Zeroth Order 44

3. 3) Inverse Propagator; First Order Correction 50

3. 4) Partial Wave Amplitudes 51

3. 5) Regge Trajectories; the Hadron Spectrum 57

3. 6) Remarks 65

$\begin{array}{lll}\text { Chapter } 4 \quad \text { Hadronic Scaling } & 67\end{array}$

$\begin{array}{ll}\text { 4. 1) Introduction } & 67\end{array}$

4.2) Six-point Conformal Amplitude $\quad 70$

4. 3) Some General Properties and the Skeleton 76 Amplitude

4. 4) Independent Correlation Model. 81 
Table of Contents - Cont'd.

Page

Chapter 5 Discussion

90

References 


\section{Chapter 1}

Introduction

Our object in this report is to study conformal symmetry as an approximate space-time symmetry of hadron systems in the high energy, large momentum transfer region. As is well known, the Poincaré group is the largest exact space-time symmetry group of physical systems. However, the conformal group, which contains the Poincaré group as one of its subgroups, has also appeared in various places as an exact symmetry group. Conformal invariance of Maxwell's equation was noticed by Bateman and Cunningham $^{1)}$ in 1909. On noticing a simple correspondence of ordinary fields over Minkowski space with fields on the four-dimensional surface in a five-dimensional projective space, manifestly conformal invariant free field equations and invariant interactions were first discussed by Dirac and Kastrup ${ }^{2}$, respectively. An attempt to formulate electrodynamics based on the conformal group was given by Page. ${ }^{3)}$ In relation to this, special conformal transformations were interpreted as transformations to constant accelerating systems. The physical interpretation of conformal symmetry and its relevance to the real world were extensively studied by warious authors, notably by Kastrup, Mack and Wess. 4) By now, the more or less accepted interpretation of special conformal transformations as local scale transformations is due to Kastrup. ${ }^{4)}$ 
A current algebraic formulation of studying broken conformal symmetry known as PCDC (partially conserved dilatation current) was given by Mack. In the framework of canonical Lagrangian field theory, it has been also shown that the conformal symmetry (or broken conformal symmetry) can be consistently formulated and also that the scale invariance necessarily implies the conformal invariance too, under very general conditions. However, recent great interest in conformal symmetry is largely due to scaling phenomena in deep-inelastic electron proton scattering predicted by Bjorken ${ }^{6)}$ and verified soon after the prediction was made by the MIT-SLAC experiment.' In an effort to explain such a peculiar behaviour of nucleon structure functions, the Wilson's operator product expansion ${ }^{8)}$ at small distances has been extended to the light cone. ${ }^{9)}$ As originally postulated by Wilson, ${ }^{8)}$ it has been assumed that the leading light-cone singularity may be determined only by scale invariance. If this is the case it is also very natural to assume conformal invariance on the light-cone based on our previous experience with local Lagrangian field theories. Conformal covariant operator product expansions have been developed by Gatto and his collaborators. ${ }^{10)}$ In a completely soluble field theory model, like the Thirring model, Wilson $^{11)}$ has shown that the scale dimensions of operators depend 
on the coupling constant. Although the results obtained from the Thirring model do not necessarily carry over to four dimensions, it is generally agreed that anomalous dimensions could play an important role in the real world (i.e. four dimensional theories as well. This belief is further strengthened by some perturbative results in renormalizable field theory models. ${ }^{13)}$ It is known that the results of the MIT-SLAC deep-inelastic electron proton scattering experiment ${ }^{7)}$ prefer at least near canonical dimensionality; the appearance of canonical scaling in ep. $\rightarrow \mathrm{eX}$ may be due to the fact that the dimension of a conserved current is canonical. However, it is true that there is no way to distinguish small deviations from the canonical dimension with present experimental accuracy. It is worth mentioning at this point that almost all calculations ${ }^{13)}$ for the anomalous dimension give very small deviation from the canonical value. In the main text we also give an estimate of the anomalous part $\delta$ for a scalar tadpole mediating the symmetry breaking. We find that $\delta$ is around 0.04 .

At this stage it is appropriate to ask the following question: how do we construct conformal invariant theory accepting the occurrence of anomalous dimensions? A big step toward this problem taken by Migdal ${ }^{14)}$ is known under the name of bootstrap approach to conformal symmetry which is essentially non-perturbative. Migdal has observed that the integral equations which determine 
three-point functions admit conformal invariant solutions and also they are free from divergences because of the presence of anomalous dimensions. The bootstrap approach has been further developed by Mack, Symanzik and Todorov. ${ }^{15)}$ A less specific but in some sense more physical approach to the problem has been made by Domokos and Kövesi-Domokos. ${ }^{16)}$ Working with infinite dimensional representation of $\mathrm{SU}(2,2)$, they have shown that conformal invariant amplitudes satisfy weak duality in the sense that they do not have simultaneous singularities in overlapping channels.

Despite all the aforementioned good features, a conformal symmetric theory cannot be a realistic model for the real world because of the discrete mass spectra of the physical particles. Therefore it is important to study the breaking of the conformal symmetry. As the title of this thesis suggests, this is one of our main themes to investigate. It will be assumed that conformal symmetry is an approximate symmetry. When the explicit symmetry breaking is turned off the following two types of limiting behaviour can occur: On the one hand, it may happen that the symmetry becomes exact, so all the masses of particles involved are zero or continuous and, of course, there shouldn't appear dimensional parameters. On the other hand, it may not be exact even in this limit. Instead, massless Nambu-Goldstone bosons ${ }^{17}$ ) appear. All the masses except the mass of such a boson remain 
$-5-$

nonzero. This latter case has been widely studied in the literature and it will motivate our work as well.

At this point it is instructive to study a simple Lagrangian field theory model due to Nambu and Freund. ${ }^{18,19)}$ Consider a Lagrangiant consisting of two scalar fields, $\varphi(x)$ and $\psi(x)$;

$$
f(x)=\frac{1}{2}\left\{\left(\partial_{\mu} \varphi\right)^{2}+(2 \mu)^{2}+\lambda_{0}(\varphi \psi)^{2}\right\}+\lambda_{1}\left(\varphi^{2}-\lambda_{2}\right)^{2}
$$

where $\lambda_{2}$ is the only dimensional parameter. We can define "the improved energy momentum tensor", $\mathbb{A}_{\mu \nu}(x)$, originally siggested by Gürsey $^{20)}$ and recently rediscovered by Calla et al . 21) With $\mathcal{H}_{\mu \nu}$ in our hand, explicit representations of the scale current $\mathrm{D}_{\mu}$ and of the special conformal currents $\mathrm{K} \mu$ are concisely written down in terms of the fields:

$$
\begin{aligned}
& D_{\mu}=x^{V} \mathscr{B}_{\mu \nu}(x), \\
& K_{\mu \nu}=\left(2 x_{\mu} x^{\rho}-g_{\mu} \rho x^{2}\right) \mathscr{B}_{\mu \nu}(x) .
\end{aligned}
$$

In the present case, however, the symmetry is broken explicitly by the last term in $\mathcal{L}(2)$, so these currents are not conserved. Let us see whether our Lagrangian admits solutions with spontaneously broken symmetry. To this end, consider the action integral

$$
W(\varphi, 4)=\int \mathcal{L}(x) d^{4} x \text {, }
$$

and take functional derivatives with respect to $\varphi$ and $\psi$, then look for constant solutions for $\frac{\delta W}{\delta \varphi}$ and $\frac{\delta W}{\delta \psi}$. We find solutions 
a) $\varphi=0, \psi=0$,
b) $\varphi=0, \psi= \pm\left(\lambda_{2}\right)^{\frac{1}{2}}$,

where a) is an unstable soiution. (This can be easily established by considering the second derivatives of the action.) Let us take the solution b) with $\psi=+\left(\lambda_{2}\right)^{\frac{1}{2}}$ and introduce a new field,

$$
x(x)=\frac{\psi^{2}(x)-\lambda_{2}}{2\left(\lambda_{2}\right)^{\frac{1}{2}}}
$$

Evidently we now have $\langle 0|\chi(x)| 0\rangle=0$. In terms of $\varphi$ and $\mathcal{X}$, the scale (conformal) breaking term in the Lagrangian is simply $-\frac{b}{2} m_{x}^{2} x^{2}$ where $m_{x}^{2}=\lambda_{1} \lambda_{2}$. In the limit of switching off the symmetry breaking $\left(\lambda_{1} \rightarrow 0\right.$ with $\lambda_{2}$ fixed $), x_{x}^{2} \rightarrow 0$ should be 22)

noted. A particularly nice feature in the case of spontaneously broken conformal symmetry is that the dynamical mechanism which gives rise to nonvanishing vacuum expectation values for $\Psi$ does not appear if we go over to the symmetry limit contrary to other types of symmetries like SU (2) $\otimes$ SU (2). The NambuFreund model is also a good example of the scalar dominance of the trace of the energy momentum tensor. In the particular model discussed here, this is easily verified by computing the trace of $G_{\mu v}$ explicitly. One finds:

$$
\oplus_{\mu}^{\mu}=\sqrt{\lambda_{2}} m_{x}^{2} x \text {. }
$$


In the main text (Sec. 3.1), a generalization of the Nambu-Freund model is found and a perturbative scheme is developed.

The plan of this report is as follows. General properties of a conformally invariant theory are treated in Chapter 2. After defining conformal transformations (Sec. 2.1), the induction procedure for the representations of SU $(2,2)$ is briefly discussed (Sec. 2.2). In Sec. 2.3 we give a plausible argument for the presence of anomalous dimensions. The concept of the effective action is discussed and it is argued that the effective action method is best suited for studying approximate symmetries of hadronic systems whose "fundamental" dynamics is unknown. Assuming asymptotic conformal symmetry, a set of diagram rules for the construction of conformal invariant irreducible vertices is given (Sec. 2.5). Some features (like the absence of ultraviolet divergences) will be briefly discussed in Sec. 2.6. Finally in Sec. 2.7, problems involving fermions will be discussed. Breaking of conformal symmetry is discussed in Chapter 3 . We give a mathematical formulation of the problem in order to put physical ideas into workable form (Sec. 3.1). Some explicit calculations are reported; the inverse propagator will be calculated explicitly to first order in the "tadpole expansion". We give explicit representations of these in momentum space (Sec. 3.2 and 3.3). We then make partial wave projections, first at integer points. Using 
Carlson's and Hartog's theorems repeatedly we prove that analytic continuation of these expansions is possible in the two relevant variables ( $l$ and $V=\tau-\sigma$ ) (Sec. 3.4). In Sec. 3.5 we extract the information about hadron resonances from the inverse propagator by looking for its zeroes. We find that hadrons lie on almost linear Regge trajectories. Their widths depend explicitly on the anomaly of the dimension, of the scalar tadpole which breaks conformal symmetry. A fit to the spectrum of the well-established baryon resonances gives a value $\delta \sim 0.04$. Further examination of the singular surfaces of the Green functions shows that at fixed angular momentum they give rise to "dilatational trajectories" as first conjectured by Del Giudice et al. ${ }^{23)}$ Chapter 3 is concludéd with some remarks. "Hadronic Scaling" is treated in Chapter 4. We calculate to zeroth order in the tadpole and use the results of the first order calculation in the spectrum; this is justified since the Brillouin-Wigner ${ }^{24)}$ perturbation method used in Ch. 3 assures the $\mathrm{n}$-th order in the "wave function" gives $\mathrm{n}+1$-th order in the eigenvalue. The necessary concepts and facts are enumerated in Sec. 4.1. The six-point conformal amplitude is considered utilizing a Mellin projection technique (Sec. 4.2). It is shown that the absorptive part of the six-point "skeleton" vanishes. This gives a natural reason for studying the perturbative calculations treated subsequently. We give a first order perturbative calculation for the absorptive 
part of the six-point amplitude. We find that the inclusive cross sections obey simple scaling law. A specific form of the scaling function is evaluated in an independent correlation model. A partonlike structure of the cross sections emerges naturally in this framework. Combining these results with the previously obtained dimensional rule we predict that at high energy and large transverse momenta the inclusive production of "heavy particles" $(\mathrm{K}, \boldsymbol{\eta}$ etc.) should be more frequent than pion production (Sec. 4. 3, 4. 4). Finally, this report is concluded with discussions in Chapter 5. 
$-10-$

Chapter 2

On Conformal Invariant Theories

2.1) Conformal Group

Even though our physical space is the four-dimensional Minkowski space we first consider the conformal group in a general n-dimensional space $\mathbf{R}_{\boldsymbol{n}}^{\mathbf{m}}$ :

$$
R_{n}^{m}=\left\{\left(x_{1}, \cdots, x_{m}, x_{m+1}, . x_{n}\right) \mid x_{i}=\operatorname{real} ;-\infty<x_{i}<\infty\right\} \text {, }
$$

with a metric tensor

$$
g_{\mu \nu}=\left\{\begin{aligned}
0 & \mu \neq \nu, \\
1 & \mu=\nu, \quad 1 \leq \mu \leq m \\
-1 & \mu=\nu, m+1 \leq \mu \leq n
\end{aligned}\right.
$$

We define conformal transformations on the space $R_{n}^{\dot{m}}$ as follows:

a) "Rotation"; $\quad x^{\prime \mu}=\Lambda^{\mu} \vee x^{\nu}$,

b) Translation; $\quad x^{\prime \mu}=x^{\mu}+t^{\mu}$,

c) Scale transformation; $\quad x^{\prime \mu}=\lambda x^{\mu}$,

d) Special conformal transformation; $\quad x^{\prime \mu}=\frac{x^{\mu}-c^{\mu} x^{2}}{1-2 c \cdot x+c^{2} x^{2}}$, where $c \cdot x=c^{\mu} x_{\mu}$. Since the above transformations constitute a $(n+2)(n+1) / 2$ parameter Lie group, it is easy to exhibit in a . standard way the Lie algebra of conformal transformations. They are: ${ }^{5), 25)}$

$\left[p^{\mu}, p^{\nu}\right]=0$,

$\left[M^{\mu \nu}, p^{\sigma}\right]=-i\left(p^{\mu} g^{\nu \sigma}-p^{\nu} g^{\mu \sigma}\right)$,

$\left[M^{\mu \nu}, M^{\sigma \rho}\right]=i\left(M^{\mu \sigma} g^{v \rho}+M^{v \rho} g^{\mu \sigma}-M^{\nu \sigma} g^{\mu \rho}-M^{\mu \rho} g^{\nu_{\sigma}}\right)$, 


$$
\begin{aligned}
& {\left[K^{\mu}, K^{\nu}\right]=0,} \\
& {\left[K^{\mu}, M^{\nu \sigma}\right]=i\left(g^{\mu \nu} K^{\sigma}-g^{\mu \sigma} K^{\nu}\right),} \\
& {\left[K^{\mu}, P^{\nu}\right]=-2 i\left(g^{\mu \nu} D+M^{\mu \nu}\right),} \\
& {\left[D, P^{\mu}\right]=-i p^{\mu},} \\
& {\left[D, K^{\mu}\right]=i K^{\mu},} \\
& {\left[D, M^{\mu \nu}\right]=0,}
\end{aligned}
$$

where $P^{\mu}, M^{\mu \nu}, K^{\mu}$ and $D$ are generators of translation, rotation, special conformal transformation and dilatation respectively. Notice that both subalgebras formed of $M^{\mu \nu}, p^{\mu}$ and $M^{\mu \nu}, K^{\mu}$ are that of $E(m, n-m)$ (pseudo-euclidean group). This fact is of some use when one induces representations of the conformal group on those of its subgroup. As we can see in Eq. (2.1) special conformal transformations have been realized nonlinearly on $R_{n}^{m}$; however, on the extended space $R_{n+2}^{m+1}$ these can be realzed linearly. We see this in a following way:

$$
\text { Define } \begin{aligned}
& J^{\mu \nu}=M^{\mu \nu} \\
& J^{n+1, \mu}=\frac{1}{2}\left(P^{\mu}-K^{\mu}\right) \\
& J^{n+2, \mu}=\frac{1}{2}\left(P^{\mu}+K^{\mu}\right) \\
& J^{n+1, n+2}=-D
\end{aligned}
$$

then simple manipulation using commutation relations Eq. (2.2) shows:

$$
\left[J^{A B}, J^{C D}\right]=i\left(g^{A D} J^{B C}+g^{B C} J^{A D}-g^{A C} J^{B D}-g^{B D} J^{A C}\right) \text {, }
$$

where $A, B, C$ and $D$ runs from 1 to $n+2$. Thus the conformal 
group is locally isomorphic to the group SO $(m+1, n-m+1)$; in fact the same relation may be shown to hold globally too. Let us now check that conformal transformations defined in Eq. (2.1) preserve the angle between two vectors in $R_{n}^{m}$. Let $x, y$ be two vectors, then the angle between them is defined by:

$$
x_{\wedge} y=\frac{x \cdot y}{\left(x^{2} y^{2}\right)^{\frac{1}{2}}} \text {. }
$$

It is now evident that translations, rotations and scale transformations preserve the angle. Note that under special conformal transformations:

$$
x \cdot y \rightarrow \frac{x \cdot y}{\sigma(x) \sigma(y)},
$$

where $\sigma(x)=1-2 c \cdot x+c^{2} x^{2}$, so $x \wedge y=x_{\wedge}^{\prime} y^{\prime}$. Consider, for instance, $n=2$, so that the conformal group is $\mathrm{SO}(3,1)$ or $\mathrm{SO}(2,2)$ depending on the metric $\mathrm{g}^{\mu \mu}(1,1)$ or $(1,-1)$. Notice that these groups are locally isomorphic to $\mathrm{SL}(2, \mathrm{C})$ and $\mathrm{SL}(2, \mathrm{R}) \otimes \mathrm{SL}(2, \mathrm{R})$, respectively. For the physical Minkowski space we have $n=4, m=1$, therefore its conformal group is $\$ O(2,4)$. One important fact is that $\mathrm{SO}(2,4)$ is homomorphic (2 to 1$)$ to the special pseudo-unitary group $\mathrm{SU}(2,2)$. This is important because we are going to deal with the covering group $\mathrm{SU}(2,2)$ instead of $S O(2,4)$. The reason for this has been discussed by Wigner $\left.{ }^{26}\right)$ for the case of the inhomogeneous Lorentz group. Wigner's 
discussion applies - with a straight-forward change of the argument to conformal groups as well. In fact, only bilinear expressions of half-integer spin fields are measureable and need to transform as single valued quantities. It is also to be noted that exact conformal symmetry necessarily implies that the mass spectrum should be either continuous or zero because of the commutation relation $\left[D, P^{\mu}\right]=-i P^{\mu}$. One should also note that the special conformal transformation can convert time-like to space-like vectors and vice versa.

2.2) Induced Representations of $\mathrm{SU}(2,2)$

Consider a four-dimensional representation of the conformal algebra expressed in terms of Dirac $\gamma$-matrices.

$$
\begin{aligned}
& P^{\mu}=-\frac{1}{2} \gamma^{\mu}\left(1+\gamma_{5}\right), \\
& M^{\mu \nu}=\frac{i}{4}\left[\gamma^{\mu}, \gamma^{\nu}\right], \\
& K^{\mu}=-\frac{1}{2} \gamma^{\mu}\left(1-\gamma_{5}\right), \\
& D=\frac{i}{2} \gamma_{5},
\end{aligned}
$$

where the $\gamma$-matrices satisfy the standard ant commutators:

$$
\left\{\gamma^{\mu}, \gamma^{\nu}\right\}=2 g^{\mu \nu} \text {. }
$$

We also have $\gamma^{0 t}=\gamma^{0}, \gamma^{k}=-\gamma^{k}$ and $\gamma^{5}=\gamma_{5}=i \gamma^{0} \gamma^{1} \gamma^{2} \gamma^{3}$, where $t$ denotes hermitian conjugate. (Note: $\gamma_{5}$ is hermitian). In order to be more specific, let us choose a particular representation of $\gamma$-matrices, namely the so-called chiral representation. They are:

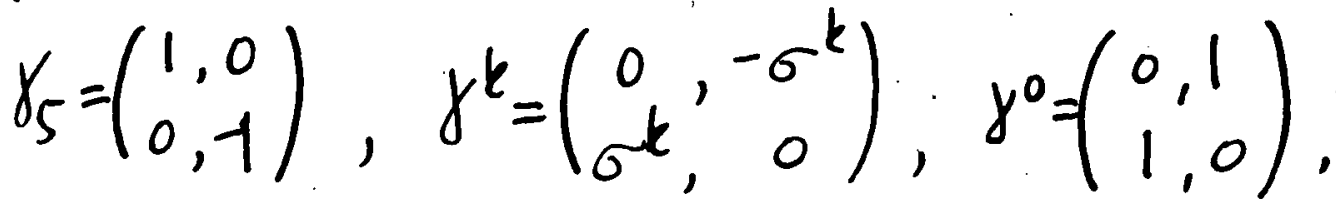


where $\sigma^{k}$ and 1 are $2 \times 2$ Pauli matrices and identity matrix respectively. It is now straight-forward to see that the following subgroups of $\mathrm{SU}(2,2):^{16)}$

$$
\begin{array}{ll}
T_{4}=\left(\begin{array}{c}
1,0 \\
t, 1
\end{array}\right), M=\left(\begin{array}{cc}
\left(m^{+}\right) & -1 \\
0, & m
\end{array}\right), \\
c_{4}=\left(\begin{array}{l}
1, c \\
0,1
\end{array}\right), \quad D=\left(\begin{array}{cc}
\lambda^{-1}, & 0 \\
0, & \lambda
\end{array}\right),
\end{array}
$$

correspond to translations, Lorentz transformations, special conformal transformations and dilatations respectively. Here $t$ and $C$ are $2 \times 2$ hermitian matrices and $m \in S L(2, C)$ and $\lambda$ is a real number. Let $\varphi(x, x)$ be a field transforming according to a representation of $\mathrm{SJ}(2,2)$ where $\mathrm{X}$ is a coordinate in Minkowski space written in a spinor form and $x$ is a Gelfand-Naimark spin label. Under $g \in \mathrm{SU}(2,2)$ :

$$
J(g) \varphi(X, x)=S(g, X, x) \varphi\left(X^{\prime}, x^{\prime}\right) \text {, }
$$

where primed variables are transformed ones. Suppose we choose $X=0$, then as is easily seen from the above Eq. (2.9), $\varphi(0, x)$ should belong to a representation of the stability group (little group) of $\mathbf{X}=0$. Note that translations are the only transformations which can shift the origin $X=0$, so the structure of the stability group is identified as $(\mathrm{SL}(2, \mathrm{C}) \otimes \mathrm{D}) \otimes \mathrm{C}$. Since we are going to induce representations of $\mathrm{SU}(2,2)$ on those of the stability group 
$(\mathrm{SL}(2, \mathrm{C}) \otimes \mathrm{D}) \otimes \mathrm{C}$, there arise two types of representations: a) Finite dimensional representations. ${ }^{27)}$ These are used for elementary particles. for example in the Lagrangian field theory such representations are assigned to the fundamental fields. b) Infinite dimensional representations. ${ }^{28)}$ Composite systems like hadrons are most likely to be described by the infinite dimensional representations even though a conformal symmetric theory does not contain any energy scale.

We further note that the representation matrices of the special conformal generators $\mathrm{K}_{\mu}$ should be null or nilpotent in the case a), which follows from the fact that the generators of translations are nilpotent in any finite-dimensional representations of the Poincaré group. So far, the most attention has been given to representations in which the generators of special conformal transformations are represented by null matrices. In what follows we also restrict ourselves to this case.

Let $\varphi(0, x)$ belong to an irreducible representation characteri zed by three complex labels $\tau, j_{1}$ and $j_{2}$ where $\tau$ is so-called conformal weight and $\left(j_{1}, j_{2}\right)$ are representation labels for $\mathrm{SL}(2, \mathrm{C})$. We have then:

$$
J(g) \varphi(0, x)=\varphi(0, x) \text { for } g \in C_{4} \text {, }
$$

and

$$
J(g) \varphi(0, x)=|\operatorname{det} g|^{\top} \alpha(g, x)^{2 j} \alpha(g \cdot x)^{2} j_{2}^{\prime} \varphi\left(0, x^{\prime}\right) \text {, }
$$

for $g \in \mathrm{SL}(2, \mathrm{c}) \otimes \mathrm{D}$ (This is known as the Weyl group, hereafter 
$-16-$

we will denote it by $W$ )

where

$$
\begin{aligned}
& x^{\prime}=\frac{g_{11} x+g_{21}}{g_{12} x+g_{22},} \\
& \alpha\left(g_{1} x\right)=g_{12} x+g_{22} .
\end{aligned}
$$

The bar here stands for complex conjugation. Under the transrations we may choose our basis such that spin indices do not change:

$J(t) \varphi(X, x)=\varphi(X+t, x)$ for $t \in T_{4}$.

Now consider $J(g) \varphi\left(X_{x}\right)$ where $g$ is a general element of $\mathrm{SU}(2,2)$ :

$J(g) \varphi(X, x)=J(g) J(X) \varphi(0, x)$

(We have $J(X) \varphi(0, x)=\varphi(X, x)$ from Eq. (2.11))

$$
=J\left(x^{\prime}\right) J(h) \varphi(0, x)
$$

where $\mathrm{X}^{\prime} \in \mathrm{T}_{4}$ and $J(h)$ stands for $J^{-1}\left(X^{\prime}\right) J(g) J(X)$ ).

We now claim that $h \in W \& \mathrm{C}_{4}$ by choosing $\mathrm{X}^{\prime}$ appropriately.

This is because we can always decompose $\mathrm{g} \in \mathrm{SU}(2,2)$ such that $\mathrm{g}=\mathrm{C}_{4} \mathrm{~W} \mathrm{~T}_{4}$. We find $\mathrm{h}$ and $\mathrm{X}^{\prime}$ explicitly as follows by straightforward calculations:

$$
\begin{aligned}
& h=\left(\begin{array}{cc}
\left(x g_{12}+g_{22}\right)^{\dagger-1}, & g_{12} \\
0 & x g_{12}+g_{22}
\end{array}\right), \\
& x^{\prime}=\left(x g_{12}+g_{22}\right)^{-1}\left(x g_{11}+g_{21}\right),
\end{aligned}
$$


$-17-$

where

$$
g=\left(\begin{array}{ll}
g_{11} & , g_{12} \\
g_{21} & , \\
g_{22}
\end{array}\right), g_{j k^{\prime} s} \text { are } 2 \times 2 \text { matrices. }
$$

The matrix $h$ can be further reduced to a product of elements in $\mathrm{W}$ and $\mathrm{C}_{4}$, namely:

$$
h=\left(\begin{array}{cc}
1, & g_{12}\left(x g_{12}+g_{22}\right)^{-1} \\
0, & 1
\end{array}\right)\left(\begin{array}{cc}
\left(x g_{12}+g_{22}\right)^{t-1}, & 0 \\
0, & x g_{12}+g_{22}
\end{array}\right)_{(2.14)}
$$

Hereafter, let us denote $\widetilde{\mathrm{h}}=\mathrm{Xg}_{12}+\mathrm{g}_{22}$, for brevity. We can now complete the induction procedure since we know how $\mathcal{J}(\mathrm{h})$ acts on $\varphi(0, x)$ from Eq. (2.10) and translation $\mathcal{J}\left(\mathrm{x}^{\prime}\right)$ on $\varphi(0, x)$.

We have therefore:

$$
\left.J(g) \varphi(X, x)=|\operatorname{det} \tilde{h}|^{2} \alpha(\tilde{h}, x)^{2 i_{1}} \overline{\alpha(\bar{h}, x}\right)^{2 i_{2}} \varphi(X, x)
$$

where $X^{\prime}$ is given in Eq. (2.13), and

$$
\begin{aligned}
& x^{\prime}=\frac{\tilde{h}_{11} x+\tilde{h}_{21}}{\tilde{h}_{12} x+\tilde{h}_{22}}, \\
& \alpha(\tilde{h}, x)=\tilde{h}_{12} x+\tilde{h}_{22} .
\end{aligned}
$$

It is known that the representations which we have just induced are unitary irreducible when the parameters $\tau, j_{1}$ and $j_{2}$ are as follows: ${ }^{29)}$

$$
\begin{aligned}
& \tau=-1+i \rho, \\
& j_{1}=-\frac{1}{2}+\frac{M}{2}+i \rho^{\prime}, \\
& j_{2}=-\frac{1}{2}-\frac{m}{2}+i \rho^{\prime},
\end{aligned}
$$


$-18-$

where $M$ is an integer and $\rho, \rho^{\prime}$ are real numbers.

If the field $\varphi(x, x)$ describes bosons, it should transform as $\tau$, $i_{1}=i_{2}=\frac{\sigma}{2} \quad$ (real). On the other hand, $\varphi(x, x)$ for fermions transform according to a reducible representation $\tau,\left(j_{1}, j_{2}\right)$ $\left(j_{2}, j_{1}\right)$. This is to accommodate parity which is a good quantum number for strong and electromagnetic interactions. Further it should be noted that finite dimensional representations are obrained when $\varphi(x, x)$ becomes a polynomial in $x, \bar{x}$.

2.3) Anomalous Dimension

As we briefly have discussed in the introductory chapter in connection with the Nambu-Freund model, in the framework of a renormalizable Lagrangian field theory it is possible to define dilatation and conformal currents introducing the conformal energy momentum tensor $\oplus_{\mu \nu}$ :

$$
\begin{aligned}
& D^{\mu}(x)=x_{\nu} \oplus \mu \nu(x), \\
& K^{\mu \nu}(x)=2 x^{\mu} x_{p} \oplus^{\nu \nu}(x)-x^{2} \oplus^{\mu \nu}(x) .
\end{aligned}
$$

Note that $\partial_{v} K^{\mu v}(x)=2 x^{\mu} \partial_{v} D^{V}(x)$, which shows that the dilatetion invariance necessarily leads to the conformal invariance. The respective generators of dilatation and special conformal transformatrons are:

$$
\begin{aligned}
& D\left(x^{0}\right)=\int x_{\nu} \mathbb{A}^{\circ \nu}(x) d^{3} x, \\
& K^{\mu}\left(x^{0}\right)=\int\left(2 x^{\mu} x_{\rho} \mathbb{A P}^{0}(x)-x^{2} \mathbb{A}^{\mu 0}(x)\right) d^{3} x .
\end{aligned}
$$


In general, $\mathrm{D}$ and $\mathrm{K}^{\mu}$ depend on time, $x^{0}$, because of symmetry breaking terms in the Lagrangian $f(x)$.

We now use canonical commutation relations:

$$
[\varphi(x), \pi(y)]_{x^{0}=y^{0}}=i \delta^{3}(x-y) \text {, }
$$

where $\pi(y)=\frac{\partial \mathcal{L}}{\partial\left(\partial^{\circ} \varphi\right)}$ is the conjugate momentum, to deduce the equal-time commutation relation between $D$ and $\varphi$ :

$$
\left[D\left(x^{0}\right), \varphi(x)\right]=\frac{1}{i}\left(x \cdot \frac{\partial}{\partial x}-d_{0}\right) \varphi(x) \text {. }
$$

Here we have to assign $d_{0}=-1$ to a scalar field, $d_{0}=-\frac{3}{2}$ to a spin $-\frac{1}{2}$ Dirac field, etc. ${ }^{30)}$ The quantity $d_{0}$ is called the canonical dimension of the field. Renormalization effects, however, destroy the canonical commutation relations in a well-known manner. In fact it is not guaranteed that we get the canonical value for the dimension of the field after renormalization, namely $d \neq d_{0}$ below:

$$
\left[D\left(x^{0}\right), \tilde{\varphi}(x)\right]=\frac{1}{i}\left(x \cdot \frac{\partial}{\partial x}-d\right) \tilde{\varphi}(x) \text {, }
$$

where $\boldsymbol{\varphi}(x)$ is a renormalized Heisenberg field. The quantity d, which is called the anomalous dimension of the field, is a function of the coupling constants contained in $\mathcal{L}$. Such an occurrence of the anomalous dimension in a renormalizable Lagrangian field theory has been first demonstrated by Wilson ${ }^{11)}$ studying the exactly soluble Thirring model, which involves a massless Dirac field with a self-coupled interaction $\lambda j_{\mu}(x) j^{\prime \mu}(x)$ where $j^{\prime \mu}(x)$ is 
the current $\bar{\psi} \gamma^{\mu} \psi$. He has shown in particular, that $d_{4}=-\frac{1}{2}-\frac{\lambda^{2}}{4 \pi^{2}}\left(1-\frac{\lambda^{2}}{4 \pi^{2}}\right)^{-1}$ for the field 4 and $\mathrm{d}=-\left(1-\frac{\lambda}{2 \pi}\right)$ $x\left(1+\frac{\lambda}{2 \pi}\right)^{-1}$ for the composite fields $\bar{\psi} \psi$ and $\bar{\psi} \gamma_{5} \psi$. This has to be compared with $d_{0}=-\frac{1}{2}$ and -1 for $\psi$ and $\bar{\psi} \psi$ (or $\bar{\psi} \gamma_{5} \psi$ ) respectively. Note $d=d_{0}$ only when $\lambda=0$, i. e. for a free field theory and also d could take any value in this specific model. Let $\boldsymbol{\lambda}$ be small and make perturbative expansion, we get:

$$
\begin{aligned}
& d_{4}=-\frac{1}{2}-\frac{\lambda^{2}}{4 \pi^{2}}+0\left(\lambda^{3}\right) \text { for } 4, \\
& d=-1+\frac{\lambda}{4 \pi}+0\left(\lambda^{2}\right) \text { for } \overline{4} 4 \text { or } \overline{4} \gamma_{5} 4 .
\end{aligned}
$$

The fact that the elementary field $\psi$ changes its scale dimension in order $\lambda^{2}$ but the composite fields like $\bar{\psi} \psi$ change dimension already in order $\lambda$ seems to be a general phenomenon in field theory. We only need to remember that similar phenomena appear in the realistic four-dimensional $\lambda \phi^{4}(x)$ moder ${ }^{3}$ there $\phi(x)$ is a scalar field.

At this point it is to be emphasized that the anomalous dimension is an inevitable concept if one wants to avoid the conformal invariant theory being a free field theory. Consider for definiteness the propagator of a spin zero (scalar) field theory. The propagator may be written:

$$
G(p)=\frac{1}{p^{2}} g\left(\frac{p^{2}}{m^{2}}\right),
$$


where $\mathrm{m}$ is the mass of the scalar field. Suppose now that the symmetry breaking is sufficiently gentle, namely $\left(H_{\mu}{ }^{\mu}\right.$ is dominated by generalized mass terms. We expect our limiting theory to be conformally invariant when $p^{2} \rightarrow \infty$. So $G(p)$ will approach a conformally symmetric propagator, say $G_{C}(p)$. As we will see in Sec. 2.5, $G_{c}(p) \propto\left(p^{2}\right)^{d}$. Therefore if $d=-1$ :

$$
G(p) \rightarrow \frac{1}{p^{2}},
$$

which implies that the limiting conformally invariant theory is a free field theory.

\section{4) Effective Action ${ }^{32)}$}

Let us attempt now to construct a phenomenological theory describing the observed hadrons. It is widely believed that hadrons are "composite" objects. This view is supported e.g. by the success of various attempts to assign excited hadron states to Regge trajectories, the fact that various "parton" models are at least qualitatively successful in explaining the main characteristics of hadronic reactions at high energies and large momentum transfers, and so on. However, neither the exact nature of the "fundamental" constituents of hadrons (if there are any) nor their dynamics are known at present. At this point it is worth remembering that in a field theoretical framework if the fundamental theory generates bound states or resonances, Haag, Nis hijma and Zimmerman ${ }^{33)}$ 
$-22-$

has shown that a local field operator could be introduced to each bound state or resonance. So let us assume that hadrons are composite objects consisting of some unknown fundamental constituents (quarks?) and furthermore that perhaps a renormalizable Lagrangian field theory exists to describe their fundamental interactions. Let $\tilde{\varphi}(x)$ be a renormalized Heisenberg field assigned to a physical hadron and $J(x)$ be the classical c-number source of $\tilde{\varphi}(x)$. It is known that the functional $Y(J)$ :

$$
Y(J)=\left\langle 0\left|T \exp \left(i \int d^{4} x \cdot J(x) \tilde{\varphi}(x)\right)\right| 0\right\rangle \text {, }
$$

is the generating functional of the time ordered vacuum expectaion values $T\left(\boldsymbol{x}_{1} \ldots \ldots \boldsymbol{x}_{h}\right)$ :

$$
T\left(x_{1}, \cdots, x_{n}\right)=\left\langle 0\left|T\left(\tilde{\varphi}\left(x_{1}\right) \cdots \tilde{\varphi}\left(x_{n}\right)\right)\right| 0\right\rangle .
$$

Note that $\mathrm{J}(\boldsymbol{x})$ is a commuting or anticommuting classical field depending on $\tilde{\varphi}(x)$ being a boson or fermion field. The connected T-functions are generated by taking functional derivatives of $X(J)$ defined by:

$$
X(J)=-i \ln Y(J) .
$$

We now define a c-number quantity $\varphi(x)$, which is known as the phenomenological or effective field of the hadron to which $\widetilde{\varphi}(x)$ is assigned, as follows:

$$
\varphi(x)=\frac{\delta X(J)}{\delta J(x)}=\frac{1}{Y(J)}\langle 0| T\left(\tilde{\varphi}(x) \exp i\left|d^{4} x J(x) \tilde{\varphi}(x)\right| 0\right\rangle
$$


$-23-$

Let us now carry out a functional Legendre transformation on $X(J)$ to eliminate $J$ in favour of $\varphi$. By doing this we get a new functional $w(\varphi)$, which is called the effective action, related to $\mathbf{X}(J)$ :

$$
W(\varphi)=X(J)-\int d^{4} x J(x) \varphi(x) .
$$

It can be shown that $\mathrm{W}(\varphi)$ is the generating functional of the one particle irreducible T-functions (also known as vertices). First let us note that $W(\varphi)$ is extremal if the external source is turned off. This can be seen from the relation:

$$
\frac{\delta W(\varphi)}{\delta \varphi(x)}=-J(x)
$$

when $J(x) \rightarrow 0$. To see more about the structure of $W(\varphi)$, we take the second functional derivatives of $\mathrm{W}$ :

$$
W_{2}\left(x, x^{\prime}\right)=\frac{\delta^{2} W}{\delta \varphi(x) \delta \varphi\left(x^{\prime}\right)}=-\frac{\delta J(x)}{\delta \varphi\left(x^{\prime}\right)} \text {, }
$$

where we have used Eq. (2.30). On the other hand the propagator (two-point Green's function) is defined by:

$$
G\left(x, x^{\prime}\right)=\frac{\delta^{2} X}{\delta J(x) \delta J\left(x^{\prime}\right)}=\frac{\delta \varphi\left(x^{\prime}\right)}{\delta J(x)} \text {. }
$$

Next consider the following integral:

$$
\begin{aligned}
& \int d^{4} x^{\prime} G\left(x, x^{\prime}\right) W_{2}\left(x^{\prime}, x^{\prime \prime}\right)=-\int d^{4} x^{\prime} \frac{\delta \varphi(x)}{\delta J(x)} \frac{\delta J\left(x^{\prime}\right)}{\delta \varphi\left(x^{\prime \prime}\right)} \\
& =-\int \delta^{4}\left(x-x^{\prime}\right) \delta^{4}\left(x^{\prime}-x^{\prime \prime}\right) d^{4} x^{\prime}=-\delta^{4}\left(x-x^{\prime \prime}\right) .
\end{aligned}
$$


This is the definition of the inverse propagator $G^{-1}$, so we get:

$$
W_{2}\left(x, x^{\prime}\right)=-G^{-1}\left(x, x^{\prime}\right) \text {. }
$$

It should be noted that $\mathrm{W}_{2}$ is no longer singular at the poles of which correspond to one-particle singularities. This is expected since the irreducible vertices do not have single particle singularities by definition. We may now proceed further by taking the third functional derivatives of $\mathrm{W}$ and find after straight-forward

$$
\begin{aligned}
& W_{3}\left(x, x^{\prime}, x^{\prime \prime}\right)=\frac{\delta^{3} W}{\delta \varphi(x) \delta \varphi\left(x^{\prime}\right) \delta \varphi\left(x^{\prime \prime}\right)} \\
& =-\int d^{4} y d^{4} y^{\prime} d^{4} y^{\prime \prime} W_{2}(x, y) W_{2}\left(x^{\prime} y^{\prime}\right) W_{2}\left(x^{\prime \prime}, y^{\prime \prime}\right) \frac{\delta^{3} X}{\delta J(y) \delta J\left(y^{\prime}\right) \delta J\left(y^{\prime \prime}\right)} .
\end{aligned}
$$

$\mathrm{W}_{3}$ is easily identified as the ordinary proper vertex. Similarly one may continue the procedure to convince oneself about the absence of the one particle singularities of the irreducible vertices. We also note that the relations between the vertices and connected $\mathrm{T}$-functions generated by $\mathrm{W}$ and $\mathrm{X}$ respectively are of the same type as those between the action integral of a classical field theory and the $\mathrm{T}$-functions in the tree approximation. This, combined with the previously noted stability property of $\mathrm{W}$ explains the names, effective action and effective field. Notice that we may now reverse the direction of the above development. Namely, by guessing some - approximate - form of W in terms of the effective fields, we may generate irreducible 
vertices. Once we accept such a purely phenomenological approach, we may discard the specific assumptions made in the beginning of this section. The only purpose was to arrive at the concept of effective actions and fields in a logical way. The quantum field theoretical framework may not exist for strong interactions, but effective actions and fields may still provide a useful phenomenological framework for hadrons. In fact, Domokos et al. ${ }^{28)}$ has shown that a consistent space-time description of hadrons is possible by introducing an infinite dimensional phenomenological field forthe hadrons.

It is clear that the symmetry properties of the effective action play an important role, since the effective action inherits the symmetries of the fundamental Lagrangian although it may possess dynamically originated symmetries as well. ${ }^{32)}$ Let $G$ be an invariance group of the Lagrangian. for definiteness we may choose $G=\mathrm{SU}(2)$ (isospin rotations). The Heisenberg fields $\widetilde{\varphi}_{\alpha}(x)$ transform as follows:

$$
J(g) \tilde{\varphi}_{\alpha}(x)=S_{\alpha \beta} \tilde{\varphi}_{\beta}(x),
$$

where $g \in \mathrm{SU}(2), \mathcal{J}(\mathrm{g})$ is the unitary operator and $\mathrm{S}$ is a representation matrix of $\mathrm{g}$. On using the assumed invariance of the Lagrangian under $G$ and Eqs. (2.27), (2.28) we can show that: 
$X\left(J^{\prime}\right)=X(J), \varphi_{\alpha}(x)_{J^{\prime}}=S_{\alpha \beta}^{-1} \varphi_{\beta}(x)_{J}$,

under the transformation of the external source,

$$
J_{\alpha}(x) \longrightarrow J_{\beta}(x) S_{\beta \alpha}
$$

It now follows from Eq. (2.37) that $\mathrm{W}$ is invariant under the transformation

$$
\varphi_{\alpha}(x) \longrightarrow S_{\alpha \beta} \varphi_{\beta}(x)
$$

With this much background we describe an ideal conformally symmetric hadronic system. One assigns an effective field $\varphi(x, x)$ not to a single hadron, but to a sequence of hadronic states - or rather, to the object to which that sequence collapses - in the conformally invariant limit and assume that effective action, $W(\varphi)$ is $S U(2,2)$ invariant. Let us make a formal functional Taylor expansion of $\mathrm{W}(\varphi)$ :

$W(\varphi)=\sum_{n} \frac{1}{n !} \prod_{i}^{n} d^{4} X_{i} \cdot d^{2} x_{i} \varphi\left(X_{i}, x_{i}\right) W_{n}\left(X_{1} x_{1}, \cdots X_{n} x_{n}\right)$, where $W_{0}=$ const, $W_{1}=0$ and $w_{2}=-G^{-1}$ etc. $\quad\left(w_{1}=0\right.$ implies the stability of the vacuum.) By the assumed invariance property of $\mathrm{W}$ under SU $(2,2)$ we have:

$$
W(J(g) \varphi)=W(\varphi) .
$$


We also assume $\tau \neq \tau_{c}$ in view of the previous discussion given in Sec. 2.3, where $\tau_{c}$ is the canonical weight of the field. From Eqs. $(2.15),(2.39),(2.40)$ we finally find the covariance condition for the $n$-point vertex $w_{n^{*}}$

$$
\begin{aligned}
& W_{n}\left(X_{1}^{\prime} x_{1}^{\prime}, \ldots, X_{n}^{\prime} x_{n}^{\prime}\right) \\
& =\prod_{i}^{n} \mid \operatorname{det} \tilde{h}\left(g_{1} X_{i}\right)^{2+\tau(i)} \alpha\left(\tau, x_{i}\right)^{2+2 j_{1}(i)} \alpha \\
& \times \frac{{ }^{2}\left(\tau, x_{i}\right)}{2+2 j_{2}(i)} W_{n}\left(X_{1} x_{1}, \ldots, X_{n} x_{n}\right),
\end{aligned}
$$

where we have used the relations:

$$
\begin{aligned}
& d^{4} X=|\operatorname{det} \tilde{h}(g X)|^{4} d^{4} X^{\prime}, \\
& d^{2} x=\alpha(\tilde{h}, x)^{2} \alpha \overline{(\tilde{h}, x)^{2}} d^{2} x /|\operatorname{det} \tilde{h}(g, X)|^{2} .
\end{aligned}
$$

Our next step is to construct the most general form of $W_{n}$ satisfying Eq. (2.41). This will be done in the next section.

2.5) Construction of Conformal Amplitudes; Diagram Rules. Following DK, we will visualize the construction of the irreducible vertices $W_{\eta}$ 's by means of a set of simple diagram rules. For the moment the effective fields $\varphi^{\tau \sigma}(X, x)$, are assumed to be of Boson type which implies $j_{1}=j_{2}=\frac{\sigma}{2}$. (We have already used this relation when writing the effective field as $\varphi^{\tau \sigma}$ ). The diagram rules for $\mathrm{W}_{n}$ are as follows:

a) Draw the simplest symmetric diagram with $n$ vertices and attach only one external line to each vertex. Thus we see that there are $n-1$ internal lines and one external line meeting at 
each vertex. For example, $n=4$;

b) Associate with each internal line, joining the vertices $j$ and $k$

Fig. 1 the propagator $\mathrm{g}_{\mathrm{jk}}$ :

$g_{j k}=\left(\frac{-\operatorname{det} X_{j k}+i o}{2}\right)^{\tau_{j k}}\left\{\left(n_{j k} \cdot X_{j k}\right)\left(\overline{n_{j k}} \cdot X_{j k}\right)\right\}^{\sigma_{j k}} N_{j k}$, where $X_{j k}^{\mu}=X_{j}^{\mu}-X_{k}^{\mu}$, and the complex lightlike vector $n_{j k}$ is given by

$$
n_{j k}^{\mu}=\left(x_{j}, 1\right) \sigma^{\mu}\left(\begin{array}{c}
\bar{x}_{k} \\
1
\end{array}\right) \text {. }
$$

The conformal weight $\tau_{j k}$ and the Lorentz label $\sigma_{j k}$ assigned to the internal line satisfy the relations which are valid by definition:

$$
\begin{aligned}
& \tau_{j k}=\tau_{k j}, \quad \tau_{j j}=0, \\
& \sigma_{j k}=\sigma_{k j}, \quad \sigma_{j j}=0 .
\end{aligned}
$$

The normalization factor Njk are chosen to be

$$
N_{j k}=\frac{1}{\Gamma\left(2+\tau_{j k}\right) \Gamma\left(1+\sigma_{j k}\right)} \text {. }
$$

c) Associate with each vertex $\mathrm{k}$ the covariance assuring factor:

$$
\delta\left(\tau_{k}-\sum_{j \neq k} \tau_{j k}\right) \delta\left(\sigma_{k}-\sum_{j \neq k} \sigma_{i k}\right)
$$


where the formal $\delta$-functions should be understood in the following sense; we observe that the labels $\tau_{j} k$ can be made to lie on a single Jordan curve in the complex $\mathcal{C}$ plane. The latter can be mapped onto the real line. Similarly for $\sigma_{j k}$. (Let $z(t)$ be a parametric equation of an arc where $t$ runs through an interval $\alpha \leq t \leq \beta$. An arc is called a Jordan curve if $Z(\alpha)=Z(\beta)$ and $Z\left(t_{1}\right)=Z\left(t_{2}\right)$ only for $\left.t_{1} t_{2}\right)$. It is also to be noted that to each external field $\varphi^{\tau_{k} \sigma_{k}}$ we have assigned its contravariant partner, $\tau_{k}^{\prime}=-\tau_{k}-2, \sigma_{k}^{\prime}=-\sigma_{k}-2$. Consider the two fields $\varphi^{\tau \sigma}, \varphi^{\tau_{\sigma}^{\prime}}$. The quantity I:

$$
I=\int d^{4} X d^{2} x \varphi^{\tau \sigma}(X, x) \varphi^{\tau^{\prime} \sigma^{\prime}}(X, x)
$$

is an invariant if $\tau+\tau^{\prime}+2=0, \sigma+\sigma^{\prime}+2=0$. Hence we call $\varphi^{-\tau-2,-\sigma-2}$ contravariant to $\varphi^{\tau \sigma}$. Eq. (2.47)can be generalized to an arbitrary number of amplitudes without any difficulty. It has been pointed out by Montvay ${ }^{34)}$ that in the construction of $\mathrm{SL}(2, \mathrm{c})$ covariants one is forced to assign contravariant fields in order to get the correct limit at the physical integer points. Our construction is a generalization of Montvays ideas to SU(2,2). d) Multiply the expression resulting from the application of rules a) through c) by an arbitrary function, $G\left(\tau_{j k}, \sigma_{j k}\right)$, of the internal labels. The function $G$ has to satisfy certain rather mild integrability conditions. In what follows, it will be assumed that $G$ is analytical around the principal series of SU(2,2). e) Integrate over the variables $\tau_{j k}, \sigma_{j k}$ along a hypercontour running through the principal series of $\mathrm{SU}(2,2)$ in each 
$-30-$

internal variable. A finite deformation of the hypercontour should be possible away from the principal series. This is because of the assumed analyticity of $G$ in the rule $d$ ).

As an illustration let us work out the four point vertex, $\mathrm{w}_{4}\left(\mathrm{x}_{1} x_{1} \ldots\right.$, $\mathrm{x}_{4} x_{4}$ ), explicitly. While we go on, we will also demonstrate the rules given here being equivalent to the rules given in DK.

Step a) is carried out in Fig. 1.

Step b):

$$
\begin{aligned}
& g_{12} g_{13} g_{14} g_{23} g_{24} g_{34} \\
& =\left(\frac{-X_{12}^{2}+i 0}{2}\right)^{\tau_{12}}\left\{\left(n_{12} \cdot X_{12}\right)\left(\bar{n}_{12} \cdot X_{12}\right)\right\}^{\sigma_{12}} N_{12} \cdots \\
& \text { Step c): }\left(\frac{-X_{34}{ }^{2}+i o}{2}\right)^{\tau_{34}}\left\{\left(n_{34} \cdot X_{34}\right)\left(\bar{n}_{34} \cdot X_{34}\right)\right\}^{\sigma_{34}} N_{34} \text {. } \\
& \delta\left(\tau_{1}-\tau_{12}-\tau_{13}-\tau_{14}\right) \delta\left(\sigma_{1}-\sigma_{12}-\sigma_{15}-\sigma_{14}\right) \therefore . . . \\
& \text {... } . \delta\left(\tau_{4}-\tau_{41}-\tau_{42}-\tau_{43}\right) \delta\left(\sigma_{4}-\sigma_{41}-\sigma_{42}-\sigma_{43}\right) \text {. }
\end{aligned}
$$

Step d) and e):

$$
\begin{aligned}
& W_{4}\left(X_{1} x_{1}, X_{2} x_{2}, X_{3} x_{3}, X_{4} x_{4}\right) \\
& =\int \cdots \int d \tau_{12} \cdots \cdot d \sigma_{34} \delta\left(\tau_{1}-\tau_{12}-\tau_{13}-\tau_{14}\right) \cdots \delta\left(\sigma_{4}-\sigma_{41}-\sigma_{42}-\sigma_{43}\right) \\
& \times G\left(\tau_{12}, \ldots, \sigma_{34}\right)\left(\frac{-X_{12}^{2}+i 0}{2}\right)^{\tau_{12}} \ldots .\left\{\left(\pi_{34} \cdot X_{34}\right)\left(\overline{r_{34}} \cdot X_{34}\right)\right\}_{34}^{\sigma_{34}} N_{34} .
\end{aligned}
$$

We have six independent $\tau_{\mathrm{jk}}$ and four $\delta$ functions, so there remain two independent parameters after taking into account $\delta$-fundtron constraints. Let us parametrize the solution of covariance equation: 
$-31-$

as follows:

$$
\begin{aligned}
& \tau_{12}=-\frac{1}{2}\left(\tau_{3}+\tau_{4}+k_{2}\right), \\
& \tau_{13}=\frac{1}{2}\left(2 \tau_{1}+2 \tau_{3}+\tau_{2}+\tau_{4}+k_{1}+k_{2}\right), \\
& \tau_{14}=-\frac{1}{2}\left(\tau_{2}+\tau_{3}+k_{1}\right), \\
& \tau_{23}=-\frac{1}{2}\left(\tau_{1}+\tau_{4}+k_{1}\right), \\
& \tau_{24}=\frac{1}{2}\left(2 \tau_{2}+2 \tau_{4}+\tau_{1}+\tau_{3}+k_{1}+k_{2}\right),
\end{aligned}
$$

where $\mathrm{k}_{1}, \mathrm{k}_{2}$ are independent channel variables introduced in DK. A particular solution when $\mathrm{k}_{1}=\mathrm{k}_{2}=0$ is denoted by $\tau_{\mathrm{jk}} 0$. It is not difficult to see that the integrations over $\tau_{\mathrm{jk}}$ reduce to

$$
\iint d k_{1} d k_{2}\left(\frac{X_{13}^{2} X_{24}^{2}}{X_{12}^{2} X_{34}^{2}}\right)^{k_{k}}\left(\frac{X_{13}^{2} X_{24}^{2}}{X_{14}^{2} X_{23}^{2}}\right)^{k_{1}} G\left(k_{1} k_{2}, \sigma_{1 k}\right)\left(\frac{-X_{12}^{2}+i 0}{2}\right)^{T_{12}{ }^{0}} \cdots\left(\frac{-X_{34}^{2}+i 0}{2}\right)^{T_{34}^{0}}
$$

omitting inessential factors. The integrations over $k_{1}, k_{2}$ can easily be recognized as Mellin representations. Similar calculaion can be done over the variables $\sigma_{\mathrm{jk}} \cdot \mathrm{w}_{4}$ may now be written in the form:

$$
\begin{aligned}
& \text { in the form: } \\
& \begin{array}{l}
W_{4}=G\left(h_{1} h_{2}, h_{1}^{\prime} h_{2}{ }^{\prime}\right)\left(\frac{-X_{12}{ }^{2}+i 0}{2}\right)^{\tau_{22}}\left\{\left(n_{12} \cdot X_{12}\right)\left(\overline{n_{12}} \cdot X_{12}\right)\right\}^{6 / 2} \\
\quad \cdots \cdots\left(\frac{-X_{34}^{2}+i 0}{2}\right)^{\tau_{34}}\left\{\left(n_{34} \cdot X_{34}\right)\left(\overline{n_{34}} \cdot X_{34}\right)\right\}^{\sigma_{34}} \cdot(2.51)
\end{array} \\
& \text { where } h_{1}, h_{2}, h_{1}{ }^{\prime}, h_{2}{ }^{\prime} \text { are independent harmonic ratios, }
\end{aligned}
$$

$$
h_{1}=\frac{X_{13}^{2} X_{24}^{2}}{X_{12}^{2} X_{34}^{2}}, h_{1}^{\prime}=\frac{\left|n_{13} \cdot X_{13}^{2}\right|\left|n_{24} \cdot X_{24}\right|^{2}}{\left|n_{12} \cdot X_{12}^{2}\right|\left|n_{34} \cdot X_{34}\right|^{2}} \text {, etc. }
$$

Equation (2.51) may be written down directly applying the rules

given in DK. This completes the demonstration. It is also 
straightforward to see that DK-representations reduce to the Symanzik ${ }^{35)}$ representations for the degenerate series of $\mathrm{SU}(2,2)$.

2. 6 Some Remarks on SU $(2,2)$ Invariant Amplitudes

As we can see from the construction rules, the two and three point vertices are special in the sense that they are determined exactly up to an arbitrary constant which depends on the conformal weights and Lorentz labels. In general we have $n(n-1) / 2$ internal weights $\tau_{\mathrm{jk}}$ and the $n$ constraints among the weights; therefore $n(n-3) / 2$ independent parameters remain.

a) Two-point vertex

In this case, $2(2-1) / 2=-1$. We have a constraint, namely, $\mathrm{w}_{2}=0$ when $\tau_{1} \neq \tau_{2}, \sigma_{1} \neq \sigma_{2}$. According to the diagram

$W_{2}\left(X_{1} x_{1}, X_{2} x_{2}\right)=\left(\frac{-X_{12}^{2}+i 0}{2}\right)^{\tau-2}\left\{\left(x_{12} \cdot X_{12}\right)\left(n_{12} \cdot X_{12}\right)\right\}(P(-\tau) P(1+\sigma))^{-1}$

where $\tau=\tau_{1}=\tau_{2}, \sigma=\sigma_{1}=\sigma_{2}$.

It should be noted that the uniqueness of $\mathrm{W}_{2}$ also follows from

the fact that it is the intertwining operator for $\mathrm{SU}(2,2)^{36)}$

b) Three-point vertex

$3(3-1) / 2-3=0$, there exists a unique solution:

$\tau_{i k}=\frac{1}{2}\left(\tau_{j}+\tau_{k}-\tau_{i}\right)$,

$\sigma_{j k}=\frac{1}{2}\left(\sigma_{j}+\sigma_{k}-\sigma_{i}\right)$.

where $(j k i)$ in cyclic order. We have for $W_{3}$ : 


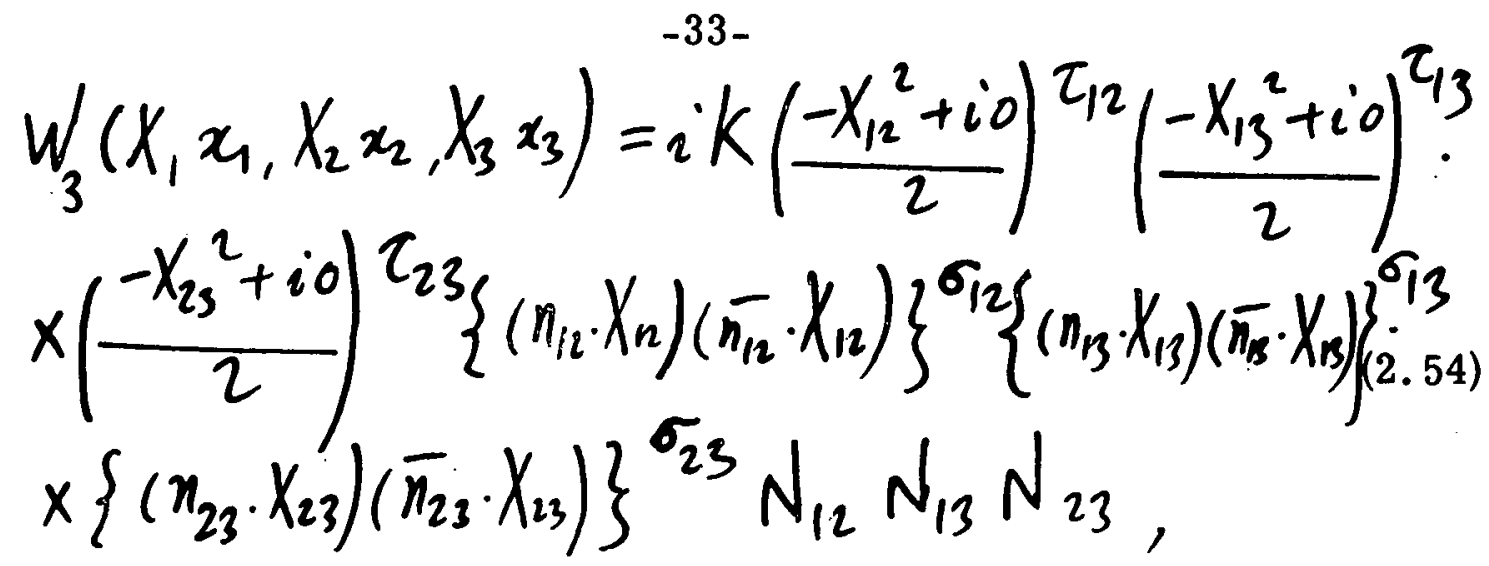

where $\mathrm{K}$ is a constant which can be determined only if we have complete solutions of dynamics. It is worthwhile to note that the fact that the conformal invariance alone could determine two and three point vertices is essentially because we cannot form conformal scalars with less than four spacetime points. ${ }^{37)}$

c) Absence of ultra-riolet divergences 14) 15) 16)

This has been first noted by Migdal ${ }^{14)}$ in a paper where he suggested a bootstrap approach to the construction of conformal invariant theory. Subsequently the absence of divergences has been further clarified by Mack and Todorov. In particular they were able to show the absence of infrared divergences too along with ultraviolet divergences. For example, in specific model of the pseudo-scalar Yukawa theory if the scale dimensions satisfy the restrictions:

$-3<d<-1$ for pseudo-scalar field, $-\frac{5}{2}<d<-\frac{3}{2} \quad$ for Dirac field, 
the complete absence of ultraviolet divergences is guaranteed. The reason behind this is roughly as follows; although propagators are more singular than the free canonical ones, but vertex functions (three-point) are less singular in such a way that they can overcompensate the singularities occurring in the propagators. Hence we have a convergent theory in the ultraviolet region.

d) Connection with duality.

An interesting property, first pointed out by Domokos and Kövesi-Domokos, ${ }^{16)}$ is that conformal covariant amplitudes do not have simultaneous singularities in the overlapping channels. It should be noted, however, that the structure of the singularities is quite different in the dual resonance amplitudes and the SU $(2,2)$ covariant ones; for instance rising Regge trajectories cannot be accommodated in the SU $(2,2)$ covariant amplitudes simply because no energy scales exist. It has been speculated that the dual resonance amplitudes may gner to the SU $(2,2)$ covariant ones when the slope of Regge trajectories becomes infinite. So far this is only a conjecture; see, however, subsequent chapters, especially $\mathrm{Ch}$. 3. The argument which led the authors quoted to the concept of weak duality goes like this; according to our Feynman-like diagram rules, especially Step a), it is quite evident that the structure 
of singularities is determined by symmetrical Feynman-like diagrams. Such a diagram is known ${ }^{38)}$ to be free of simultaneous singularities in the overlapping channels. A fur ther connection with duality has been given by Del Giudice et al. ${ }^{23)}$ in a different context introducing the concept of dilatation trajectories.

\section{7) Problems with Fermions}

In this section we briefly discuss how to modify our rules in order to construct SU $(2,2)$ covariant amplitudes including fermions. First, let us consider a spin-1/2 Dirac field, $\psi(x)$. It is easily seen that the conformal invariant Dirac propagator is given by ${ }^{15)}$

$$
P\left(\frac{5}{2}+d\right)^{-i} \gamma^{\mu} \partial_{\mu}\left(x^{2}-i 0\right)^{d+\frac{1}{2}} \text {, }
$$

where $\mathrm{d}$ is a scale dimension of $\psi$ and $\partial_{\mu}=\frac{\partial}{\partial x^{\mu}}$. We have also chosen a normalization factor according to our rule. When the dimension, $d$ becomes canonical, $(d=-3 / 2)$, the propagator (2.56) reducesto that of a free field theory. Notice that the factor $\left(x^{2}-i 0\right)^{d+1 / 2}$ is just a conformal invariant propagator of a scalar field with a scale dimension, $d+1 / 2$. In general it can be shown that the conformal invariant amplitudes containing fermions reduce essentially to those of bosons. This is evident in the case of propagators as we see from Eq. (2.56). In order to understand how the rules should change, we consider a three point function 
as an example. Suppose the vertex number 1 and 2 are attached to the Dirac field, $\psi$, and a scalar field, say $\phi$, is attached to the vertex number 3 . On remembering the conservation law of "particles of half-integer spin", we find that there are only two ways of satisfying this;

a) the internal line connecting vertices 1 and 2 should carry a spin-1/2 quantum number, or (and), b) the internal lines connecting vertices 1 and 3,3 and 2 are fermion lines. The case a) corresponds to a coupling $\bar{\psi} \psi$. On the other hand, if the theory contains both $\bar{\psi} \psi \phi$ and $\phi^{3}$ couplings, then we have to take into account the cases a) and b) together in constructing an amplitude. The diagram rules are now simply, a) Associate the fermion propagator with each internal fermion line. b) Make sure that dimensions are conserved at each vertex. c) Add all possible diagrams permitted after the selection rules (or conservation laws) have been applied.

One must also notice that the above considerations can be carried over to infinite dimensional representations of SU $(2,2)$ about which we are presently concerned at least in one possible version of the theory. Namely, in the framework of a relativistic orbital excitation model for hadrons ${ }^{39)}$ it is assumed that the spin and orbital degrees of freedom are decoupled in the lowest order approximation. The propagators in such a theory are simply obtained by multiply- 
ing the spin part of the propagators with the orbital part. Since the orbital part of the propagator is well described by the boson propagator, it is easily seen that the fermion propagator is given by:

$$
\gamma_{\mu} \frac{\partial}{\partial x_{\mu}} g
$$

where $\mathrm{g}$ is a boson propagator already given in Eq. (2.57).

It is to be remarked at this point that even though this is not the most general theory, it is certainly one of the simplest possible theories.

Now the modification of diagram rules given in Sec. 2.5 necessary in order to accommodate fermions is straight-forward;

Rule $b^{1}$ )With each fermion internal line we associate the propagator:

$$
S_{j k}=\gamma^{\mu} \frac{\partial}{\partial x_{j k}^{\mu}} g_{j k},
$$

where $\gamma^{\mu}$ are Dirac matrices.

Rule $C^{1}$ ) At each vertex the conservation law of particles of halfinteger spin has to be taken into account; the Dirac indices carried by fermion lines have to be coupled to invariants. 


\section{Chapter 3}

\section{Broken Conformal Symmetry}

\section{1) Formulation of the Problem}

We have seen many theoretically remarkable features of the conformal invariant theory in the preceding chapter. However it is undérstandable that we have not gained much practically unless we have a definite scheme to break the conformal symmetry down in the real world. The zero (or continuous) mass spectra are simply not realized in nature. Evidently, nature does not choose manifest conformal symmetry. However it is perfectly possible and also very reasonable intuitively - to expect the symmetry to show up as an approximate space time symmetry in some kinematical region. Now the question is "which region?". At present we only have a highly speculative answer; the small distance region, or, equivalently, the high energy, large momentum transfer regions of reaction amplitudes are the most likely candidates.

The behaviour of field theories at small distances has been extensively investigated. Note that in this region we are dealing with completely off-shell amplitudes, hence, it seems that the knowledge of the short distance limit of a theory is of no direct relevance to the observed amplitudes. We want to assume conformal symmetry as an approximate symmetry of the strong interactions which show up as an exact one at short distances. 
In order to establish a connection with observable amplitudes, it is, however, necessary to discuss the possible mechanisms by which this asymptotic symmetry is broken. This is the subject of this chapter. In the introductory chapter, we have briefly described the well-known Nambu-Freund model involving two scalar fields. There we have noted that manifest conformal symmetry is broken by a non-vanishing vacuum expectation value of a composite scalar field $X(x)$ of scale dimension equal to -2 . The Nambu-Freund mechanism provides an explicit example for a "gentle" breaking of SU $(2,2)$. We wish now to generalize the Nambu-Freund model to study the hadron spectrum. Our results, however, reflect very few of the specific features of the model; therefore we believe that they are more general than the context in which they are derived.

In the subsequent calculations, we will be dealing with Bosons only. The modifications required to accommodate Fermions are trivial in view of our discussion in Sec. 2.7 (see, however, Sec. 3.6). Assuming that we have two kinds of Bosons, $\varphi^{\tau \sigma}(X, x)$ and $\psi(X)$, we guess the form of an effective action $w\left(\varphi^{2 \sigma}, \psi\right)$ in such a way that it reduces to the familiar Nambu-Freund model if both $\varphi$ and $\psi$ are canonical scalar fields. In the conformal limit the field $\varphi^{\tau \sigma}$ describes a "collapsed tower" of hadrons and $\psi$ is assumed to be a scalar of the scale dimension $d \sim-1$. Note that we are allowing the appearance of an anomaly in dimen- 
$-40-$

sion. We now specify our model as follows:

$$
\begin{aligned}
& W\left(\varphi^{\tau \sigma}, \psi\right) \\
& =W\left(\varphi^{\tau \sigma}\right)+W(\varphi)+W_{\text {int }}\left(\varphi^{\tau \sigma}, \psi\right)+W^{\prime}(\psi) .
\end{aligned}
$$

where the functionals $\mathrm{w}\left(\varphi^{\tau \sigma}\right)$ and $\mathrm{w}(\psi)$ are given by a functonal Taylor series as in Eq. (2.39). It is to be noted that in the construction of $\mathrm{W}(\psi)$ there is a slight modification of the ciagram rules ${ }^{16)}$ because of the fact that we do not have spin variables, $\mathfrak{X}_{\boldsymbol{k}}$, to integrate over. With such a modification in mind we find for instance:

$$
\begin{aligned}
& W(\psi)=\text { cost. }+\int d^{4} X d^{4} Y \psi(X) \psi(Y) . \\
& x\left(\frac{-\operatorname{det}(X-Y)+i \delta}{2}\right)^{-3-\delta} \Gamma(-1-\delta)^{-1}+O\left(\psi^{4}\right),
\end{aligned}
$$

where $\delta$ is the anomalous part of the scale dimension of $\psi$. We choose $\mathrm{W}_{\text {int }}$ as follows:

$$
\begin{aligned}
W_{\text {int }}\left(\varphi^{\tau \sigma}, \psi\right) & =\int d^{4} X_{1} d^{2} x_{1} d^{4} X_{2} d^{2} x_{2} d^{4} X_{3} \varphi^{\tau}\left(X_{1} x_{1}\right) \varphi^{\tau}\left\{X_{2}, x_{2}\right) \\
& \times F\left(X_{1} x_{1}, X_{2} x_{2} ; X_{3}\right) \psi^{2}\left(X_{3}\right)+\cdots,
\end{aligned}
$$

where $F$ is nothing but a SU $(2,2)$ covariant three point vertex which may be given by Eq. (2.54) with a simple change (ie. $\left.\tau_{3} \rightarrow \tau_{3}+2\right)$ because of $\psi$ being the scalar field. We find a unique solution for $\tau_{j k}, \sigma_{j k}$ :

$$
\tau_{12}=\frac{1}{2}\left(-\tau_{1}-\tau_{2}+d\right)
$$


$-41-$

$$
\begin{aligned}
& \tau_{2 s}=\frac{1}{2}\left(\tau_{1}-\tau_{2}-d\right)-2, \\
& \tau_{13}=\frac{1}{2}\left(-\tau_{1}+\tau_{2}-d\right)-2,
\end{aligned}
$$

and $\sigma_{12}=\sigma, \sigma_{13}=\sigma_{23}=0$

where $d$ is the scale dimension of the composite field $\psi^{2}$.

Therefore $F$ is now given by:

$$
\begin{aligned}
& F\left(X_{1} x_{1}, X_{2} x_{2} ; X_{3}\right)=i K\left(\frac{-\operatorname{det} X_{12}+i 0}{2}\right)^{\frac{1}{2}\left(-\tau_{1}-\tau_{2}+d\right)}\left\{\left(\pi_{12} \cdot X_{12}\right)\left(\bar{n}_{12} \cdot X_{n}\right)\right\}^{\sigma} \\
& \times\left(\frac{-\operatorname{det} X_{23}+i 0}{2}\right)^{\frac{1}{2}\left(\tau_{1}-\tau_{2}-d\right)-2}\left(\frac{-\operatorname{det} X_{15}+i 0}{2}\right)^{\frac{1}{2}\left(-\tau_{1}+\tau_{2}-d\right)-2} \\
& \times\left\{P(1+\sigma) P\left(2+\frac{1}{2}\left(-\tau_{1}-\tau_{2}+d\right)\right) P\left(\frac{1}{2}\left(\tau_{1}-\tau_{2}-d\right)\right) P\left(\frac{1}{2}\left(-\tau_{1}+\tau_{2}-d\right)\right\}^{-1} .\right.
\end{aligned}
$$

Finally $\mathrm{W}^{\prime}$ is chosen: as follows:

$$
W^{\prime}(\psi)=\lambda \int d^{4} x\left(\frac{d}{\psi}\left(\psi^{2}(x)\right)^{-\frac{4}{2}}+\left(m^{2}\right)^{2+\frac{d}{2}} \psi^{2}(x)\right)
$$

where $\lambda$ is the dimensionless coupling constant and $m$ is a mass parameter. The action $\mathrm{W}^{\prime}$ contains an explicit symmetry. breaking term. This is necessary for the study of the spontaneously broken space-time symmetry as we have noted in Ch. 1. In order to see whether our model admits nonvanishing vacuum expectation values we have to look for constant solutions of $\frac{\delta W}{\delta \varphi^{\tau} \sigma}$
$\delta W$ and $\frac{\delta W}{\delta \psi}$. Indeed we find two solutions:

a) $\varphi^{\tau \sigma}=0, \psi=0$,

b) $\varphi^{26}=0, \psi^{2}=\left(m^{2}\right)^{-\frac{d}{2}}$. 
$-42-$

Evidently the solution b) is what we want. Let us now define a new field $\chi$ essentially by "shifting the origin of $\psi^{2}$-space":

$$
x(x)=\left(m^{2}\right)^{-\frac{d}{4}} \cdot\left(\left(m^{2}\right)^{\frac{d}{2}} \psi^{2}(x)-1\right) \text {. }
$$

One must notice the complete similarity of our $\mathcal{X}$-field to the Nambu-Freund $\mathcal{X}$ introduced in $\mathrm{Ch}$. 1. Notice also that the vacuum expectation value of $\mathcal{X}$ is zero. On rewriting $\mathrm{W}$ in terms of $\mathcal{X}$, we first of all find that $w^{\prime}$ can be expanded into a series in powers of $\mathcal{X}$. It is to be noticed that this is not possible for $W^{\prime}$ in terms of the field $\psi$. We have:

$$
\begin{aligned}
W^{\prime} & =\lambda m^{4} \int d^{4} X\left\{\frac{d}{4}\left(1+\left(m^{2}\right)^{\frac{d}{4}} x(x)\right)^{-\frac{4}{d}}+\left(1+\left(m^{2}\right)^{\frac{d}{4}} x(X)\right)\right\} \\
& =\text { comet. }+\frac{\lambda}{2}\left(m^{2}\right)^{\frac{d}{2}}\left(1+\frac{4}{d}\right) \int d^{4} X x^{2}(X)+\ldots
\end{aligned}
$$

We also find that $W_{\text {int }}$ can be written in the form:

$$
W_{\operatorname{int}}\left(\varphi^{\tau \sigma}, x\right)=W_{\text {int }}^{\prime}\left(\varphi^{\tau \sigma}, x\right)+W_{\text {int }}^{\prime \prime}\left(\varphi^{\tau \sigma}\right)
$$

where $\mathrm{W}_{\text {int }}^{\text {"I }}$ represents a series of "tadpole terms":

$$
\begin{aligned}
& \text { Went }{ }^{\prime \prime}\left(\varphi^{c \sigma}\right)=\left(m^{2}\right)^{-\frac{d}{2}} \int d^{4} X_{1} d^{2} x_{1} d^{4} X_{2} d^{2} x_{2} d^{4} X_{3} \\
& \times \varphi^{2 \sigma}\left(X_{1} x_{1}\right) \varphi^{2 \sigma}\left(X_{2} x_{2}\right) F\left(X_{1} x_{1}, X_{2} x_{2} ; X_{3}\right)+\ldots . .
\end{aligned}
$$

From Eqs. (3.1), (3.2), (3.9), (3.10) it is easily seen that, for example, the inverse propagators of the field $\varphi^{\mathrm{C \sigma}}$ are given 
as follows:

$$
\begin{aligned}
& G^{-1}\left(x_{1} x_{1}, x_{2} x_{2} ; \tau_{1} \tau_{2} \sigma\right) \\
& =-\delta\left(\tau_{1}-\tau_{2}\right) w_{2}\left(x_{1} x_{1} x_{2} x_{2} ; \tau \sigma\right)-\left(m^{2}\right)^{-\frac{d}{2}} \int d^{4} x_{3} F\left(x_{1} x_{1}, x_{2} x_{2} ; x_{3}\right)
\end{aligned}
$$

+ (higher order terms in the tadpole expansion),

where $\mathrm{W}_{2}$ is the $\mathrm{SU}(2,2)$ covariant two-point vertex already given in Sec. 2.6. Two important facts should be noticed immediately trom the Eq. (3.11).

a) The symmetry breaking causes a dimensional mixing; the Green's functicn of the field $\varphi^{\tau \sigma}$ is no longer diagonal in the conformal quantum numbers, $\tau, \sigma$. However in the model being studied here there is no mixing in the Lorentz label, $\sigma$, to first order in the symmetry breaking.

b) The $\mathrm{SU}(2,2)$ limit of the theory determines the two-point vertex, $-G^{-1}$, uniquely to first order in the symmetry breaking; the arbitrary constant $\mathrm{K}$ in the $\mathrm{SU}(2,2)$ covariant three-point function can be absorbed into the mass scale of $\mathrm{m}$.

It is also to be noted that our model indeed reduces to the NambuFreund model. This is most easily accomplished through the use of Montvay's expression of $\varphi^{\tau \sigma}(X, x)$ in terms of a field in the canonical basis and of the relation: 
$-44-$

$\lim _{v \rightarrow-n} \frac{2^{3(v+1)}\left(\frac{-x^{2}+i n}{2}\right)^{v}}{P(2+v)}=\frac{2 \pi^{2}}{(n-1) ! i} \square^{n-2} \delta^{4}(x)$

where $n=2,3,4, \ldots ., \square=\frac{\partial}{\partial x^{\mu}} \frac{\partial}{\partial x_{\mu}}$. Notice that the right-hand side of the Eq. (3.12) is essentially the residue of $(-\operatorname{det} \mathrm{X}+\mathrm{io})^{\nu}$ at $\nu=-\mathrm{n}$.

3.2) Inverse Propagator; Zeroth Order

As one car see from Eq. (3.11) we only need to calculate $\mathrm{W}_{2}$ in the zeroth order. In a graphical notation we have:

$$
W_{2}=x_{2}, x_{2}, x_{1}
$$

Fig. 2

The representation in the coordinate space is given by Eq. (2.52).

Let us now Fourier-transform $\mathrm{W}_{2}$ :

$$
W_{2}\left(P, x_{1} x_{2} ; \tau \sigma\right)=\int d^{4} X_{12} \cdot W_{2}\left(X_{1} x_{1}, X_{2} x_{2} ; \tau \sigma\right) e^{i P \cdot X_{12}},
$$

where the momentum $P$ is written in a spinor form, namely, $P=p^{\mu} \sigma_{\mu}$. We note that the Fourier transform can be readily carried out when $\sigma=0$, i. e. spinless particles:

$$
\left(\frac{-p^{2}-i 0}{2}\right)^{\tau} P(2+\tau)^{-1}=\frac{1^{1}}{(2 \pi)^{2}} \int d^{4} x\left(\frac{-x^{2}+i 0}{2}\right)^{-2-\tau} \Gamma(-\tau)^{-1} e^{i p \cdot x}
$$

where $\tau \rightarrow \tau+2$ should be made. In our subsequent calculations, 
$-45-$

we also use the following relation frequently:

$$
Z^{\lambda}=\frac{i^{\lambda \lambda}}{\Gamma(-\lambda)} \int_{0}^{\infty} \frac{d \alpha}{\alpha} \alpha^{-\lambda} e^{-i \alpha z},
$$

which is valid for $I_{m} z ? n$. For other values of $z$ the anallytic continuation is assumed. Let us now write down an integral representation of $\mathrm{w}_{2}$, using the relation, Eq. (3.15), we obtain:

$$
W_{2}\left(p, x_{1} x_{2} ; \tau \sigma\right)=\frac{2^{\tau+2}(-i)^{\tau-\sigma+2}}{P(1+\sigma) p(-\sigma) p(2+\tau) P(-\tau)} \int_{0}^{\infty} d \alpha d \beta \alpha^{\tau+1} \beta^{-\sigma-1} \text {. }
$$

$x \int d^{4} X \exp -i\left\{-\alpha X^{2}+\beta(n \cdot X)(\bar{n} \cdot X)+p \cdot X\right\}$

where the integrand always includes the usual damping factor $\exp -\epsilon(\alpha+\beta), \epsilon>0$, which, however, will not be written out explicitly from now on. It is to be noticed that $(n \cdot X)(\bar{n} \cdot X)$ is a real number. At this point we introduce a real symmetric second rank tensor $\mathrm{A}_{\mu \nu}$ :

$$
A_{\mu \nu}=-\alpha g_{\mu \nu}+\beta \frac{1}{2}\left(n_{\mu} \bar{n}_{\nu}+n_{\nu} \bar{n}_{\mu}\right) \text {, }
$$

then:

$$
X^{\mu} A_{\mu \nu} X^{\nu}=-\alpha X^{2}+\beta(n \cdot X)(\bar{n} \cdot X) \text {. }
$$

Recalling that $n, \bar{n}$ are complex light-like vectors, we introduce two more real light-like vectors $\mathrm{k}, 1$ :

$$
k^{\mu}=\left(x_{1}, 1\right) \sigma^{\mu}\left(\begin{array}{c}
\bar{x}_{1} \\
1
\end{array}\right), \ell^{\mu}=\left(x_{2}, 1\right) \sigma^{\mu}\left(\begin{array}{c}
\bar{x}_{2} \\
1
\end{array}\right) \text {. }
$$


$-46-$

42)

A standard spinor calculus gives us the following identity:

$$
n_{\mu} \bar{n}_{\nu}+n_{\nu} \bar{n}_{\mu}=-(k \cdot 2) g_{\mu \nu}+k_{\mu} l_{\nu}+l_{\mu} k_{\nu} \text {. }
$$

So we now have:

$$
A_{\mu \nu}=-\left(\alpha+\frac{\beta}{2}(k \cdot l)\right) g_{\mu \nu}+\frac{\beta}{2}\left(k_{\mu} l_{\nu}+l_{\mu} k_{\nu}\right) \text {. }
$$

It is easily seen that the matrix $\mathrm{A}_{\mu}$ has two distinct eigenvalues, each doubly degenerate :

$-\alpha,-\alpha-\frac{\beta}{2}(k \cdot l)$.

These are negative definite, since the time component of the light -like vectors $k$, : is shown to be positive; in fact $k^{0}=\left|x_{1}\right|^{2}+1$, $\ell^{0}=\left|x_{2}\right|^{2}+1$. Let $\mathrm{q}$ be a particular vector orthogonal to $\mathrm{k}, 1$ and $r^{\mu}=\epsilon^{\mu \nu \rho \lambda} k_{\nu} l_{\rho} q_{\lambda}$, then we see that the vectors $k, 1, q, r$ are eigenvectors of $A$ :

$$
\begin{aligned}
& A_{\mu}{ }^{V} k_{\nu}\left(\text { or } l_{\nu}\right)=-\alpha k_{\mu}\left(\text { or } l_{\mu}\right), \\
& A_{\mu}{ }^{\nu} q_{\nu}\left(\text { or } r_{\nu}\right)=-\left(\alpha+\frac{\beta}{2}(k \cdot l)\right) q_{\mu}\left(\text { or } r_{\mu}\right) .
\end{aligned}
$$

Accordingly:

$\operatorname{det} A_{\mu}{ }^{V}=\alpha^{2}\left(\alpha+\frac{\beta}{2}(k \cdot l)\right)^{2}$.

The inverse of the matrix $A$ is found to be: 
$-47-$

$$
A_{\mu \nu}^{-1}=\frac{-1}{\alpha\left(\alpha+\frac{\beta}{2}(k \cdot l)\right)}\left\{\alpha g_{\mu \nu}+\frac{\beta}{2}\left(k_{\mu} l_{\nu}+k_{\nu} l_{\mu}\right)\right\} .
$$

We are now able to carry out the $\mathrm{X}$-integration:

$$
\begin{aligned}
\dot{W}_{2} & =\frac{-2^{\tau+2}(-i)^{\tau-\sigma}}{P(1+\sigma) P(-\sigma) P(2+\tau) P(-\tau)} \int d \alpha d \beta \alpha^{\tau+1} \beta^{-\sigma-1} \int d^{4} X e^{i\left(x^{\mu} A_{\mu \nu} X^{\nu}+P \cdot X\right)} \\
& =" \quad " \quad " \quad \int d \alpha d \beta \alpha^{\tau+1} \beta^{-\sigma-1}(\operatorname{det} A)^{-\frac{1}{2}} e^{-\frac{l^{\prime}}{4} P^{\mu} A_{\mu \nu}^{-1} P^{\nu}}
\end{aligned}
$$

Putting Eqs. (3.23), (3.24), (3.25) together we get:

$$
\begin{aligned}
W_{2}= & \frac{i^{\tau+2}(-i)^{\tau-\sigma+1} \pi^{2}}{\Gamma(1+\sigma) p(-\sigma) P(2+\tau) \Gamma(-\tau)} \int_{0}^{\infty} d \alpha d \beta \frac{\alpha^{2} \beta^{-\sigma-1}}{\alpha+\frac{\beta}{2}(k \cdot l)(3.26)} \\
& x \exp \frac{1}{4 \alpha\left(\alpha+\frac{\beta}{2}(k \cdot l)\right)}\left(\alpha p^{2}+\beta(p \cdot k)(p \cdot l)\right) .
\end{aligned}
$$

Let us introduce a variable $\vartheta$ which will turn out to be the angle between the two light-like vectors $\mathrm{k}^{\mu}$ and $1^{\mu}$ in the rest frame of the momentum P:

$$
\cos \theta=1-p^{2} \frac{(k \cdot l)}{(p \cdot k)(p \cdot l)}
$$

After substituting a variable $\gamma=\beta(k \cdot l) / 2$, we find:

$$
\begin{aligned}
& W_{2}=\frac{2^{\tau+2}(-i)^{\tau-\sigma+1} \pi^{2}}{P(1+\sigma) P(-\sigma) P(2+\tau) P(-\tau)}\left(\frac{k \cdot l}{2}\right)^{\sigma} \int_{0}^{\infty} d \alpha d \gamma . \\
& x \frac{\alpha^{2} \gamma^{-\sigma-1}}{\alpha+\gamma} \exp \frac{i p^{2} \operatorname{cosec}^{2} \frac{\theta}{2}}{4 \alpha(\alpha+\gamma)}\left(\gamma+\alpha \sin ^{2} \frac{\theta}{2}\right) .
\end{aligned}
$$

Again we change variables $t=\alpha^{-1}, u=\gamma t$, and it turns out that the integration over $t$ can be easily evaluated. So we have now: 
$-48-$

$$
\begin{aligned}
& W_{2}=\frac{2^{\tau+2}(-i)^{\tau-\sigma+1} \pi^{2} p(\sigma-\tau)}{p(1+\sigma) p(-\sigma) P(2+\tau) P(-\tau)}\left(\frac{k \cdot l}{2}\right)^{\sigma}\left(\frac{-i p^{2}}{4}\right)^{\tau-\sigma} . \\
& \quad \times\left(\sin ^{2} \frac{\theta}{2}\right)^{\sigma-\tau} \int_{0}^{\infty} d u i^{-\sigma-1}(1+u)^{\sigma-\tau-1}\left(u+\sin ^{2} \frac{\theta}{2}\right)^{\tau-\sigma} .
\end{aligned}
$$

The integral is easily recogni zed as one of standard integral representations of the hypergeometric function, $2 F_{1}$ :

$$
\begin{aligned}
& { }_{2} F_{1}(a, b ; c ; 1-2) \\
& =\frac{1}{B(u, c-u)} \int_{0}^{\infty} d u u^{c-b-1}(1+u)^{a-c}(u+2)^{-a},
\end{aligned}
$$

where $B$ is a beta-function. So we get an explicit momentum space representation of $\mathrm{W}_{2}$ :

$$
\begin{aligned}
& W_{2}=\frac{2^{\tau}(2 \pi)^{2}}{P(2+\tau) P(-\tau) i} P(\sigma-\tau)\left(\frac{k \cdot l}{2}\right)^{\sigma}\left(-\frac{p^{2}}{4}\right)^{\tau-\sigma} . \\
& \quad \times\left(\sin ^{2} \frac{\theta}{2}\right)^{\sigma-\tau} F_{1}\left(\sigma-\tau, \sigma+\mid ; 1 ; \cos ^{2} \frac{\theta}{2}\right) .
\end{aligned}
$$

It is easily checked that we recover Eq. (3.14) from Eq. (3.31)

when $\sigma=0$. Equation (3.31) can be written in the form containing a Jacobi function:

$$
\begin{aligned}
& \text { ing a Jacobi function: } \\
& \begin{array}{l}
W_{2}=\frac{2^{6}(2 \pi)^{2}}{P(2+\tau) P(-\tau) i} P(\sigma-\tau)\left(\frac{k \cdot l}{2}\right)^{\sigma}\left(\frac{-P^{2}}{2}\right)^{\tau-\sigma} \\
\quad x\left(\sin ^{2} \frac{\theta}{2}\right)^{\sigma-\tau} P_{\tau-\sigma, 44)}^{(0,2 \sigma-c)}(-\cos \theta),
\end{array} \\
& \text { where we have used the relation: }
\end{aligned}
$$

$$
P_{n}^{(\alpha, \beta)}(x)=(-1)^{n}\left(\begin{array}{c}
n+\beta \\
n
\end{array}\right){ }_{2} \bar{r}_{1}\left(\begin{array}{c}
n, n+\alpha+\beta+1 ; \\
\left.\beta+1 ; \frac{1}{2}(1+x)\right) .
\end{array}\right.
$$


$-49-$

Notice, under dilatation $p \rightarrow \lambda P$, that $w_{2} \rightarrow \lambda^{2(\tau-\sigma)} w_{2}$ which assigns the scale dimension $\sigma-\tau$ to the field $\varphi^{\tau \sigma}$. The cut of $\mathrm{W}_{2}$ should be chosen to lie along the positive real axis. Even though Eqs. (3.31), (3.32) are our final expressions of the Fourier transformed $\mathrm{w}_{2}$, it is necessary to derive a different expression of $\mathrm{W}_{2}$ in order to avoid similar long calculations of the Fourier transformed representation in the following section. This time, we start from the representation, Eq. (3.14) and then make use of the relation, Eq. (3.15). After some straight-forward manipulations we find:

$$
\begin{aligned}
& W_{2}\left(P, x_{1} x_{2} ; \tau \sigma\right) \\
& =\frac{2^{-\tau}(2 \pi)^{2}}{P(1+\sigma) P^{2}(-\sigma) P(2+\tau) i} \int_{0}^{\infty} d \alpha d \beta(\alpha \beta)^{-\sigma-1}\left(-P^{2}-i 0\right)^{\tau},
\end{aligned}
$$
where $\mathbb{P}=P+\alpha n-\beta \bar{n}$ is a complex four vector. Equation (3.34) is defined in the region, $\operatorname{Im} n^{\circ}>0, \operatorname{Im} n^{k}<0$.

Beyond this region it is defined by the analytic continuation. Let us now compare two expressions, Eqs. (3.32) and (3.34). We obtain:

$$
\begin{aligned}
& \frac{1}{P(1+\sigma) p^{2}(-\sigma) 2^{\sigma}} \int_{0}^{\infty} d \alpha d \beta(\alpha \beta)^{\sigma-1}\left(-p_{-i}^{2} \cdot \tau\right)^{\tau} \\
& =\frac{2^{2}}{\Gamma(-\tau)} \Gamma(\sigma-\tau)\left(\frac{k \cdot l}{2}\right)^{\sigma}\left(-\frac{p^{2}}{2}\right)^{\tau-\sigma}\left(\sin ^{2} \frac{\theta}{2}\right)^{\sigma-\tau} p_{\tau-\sigma}^{(0,2 \sigma-\tau)}(-\cos \theta)
\end{aligned}
$$


$-50-$

3.3) Inverse Propagator; First Order Correction

In our symmetry breaking scheme the first order correction term to the inverse propagator is given by the second term of Eq. (3.11). In a graphical notation:

$$
W_{2}^{\prime}=
$$

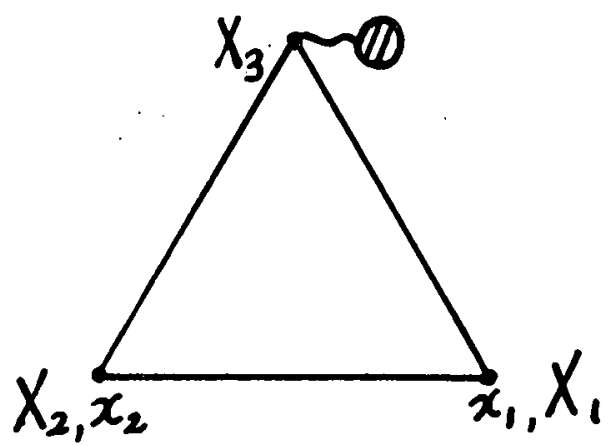

In what follows, the Fourier transform of $w_{2}^{\prime}\left(=\left(m^{2}\right)^{-\frac{d}{2}} \int d^{4} x_{3}\right.$ $\left.F\left(X_{1} x_{1} X_{2} x_{2} ; X_{3}\right)\right)$ will be given explicitly along the line which we followed to derive Eqs. (3.34), (3.35). After a somewhat tedibus calculation we find:

$$
\begin{aligned}
& W_{2}^{\prime}\left(p, x_{1} x_{2} ; \tau_{1} \tau_{2} \sigma\right)
\end{aligned}
$$

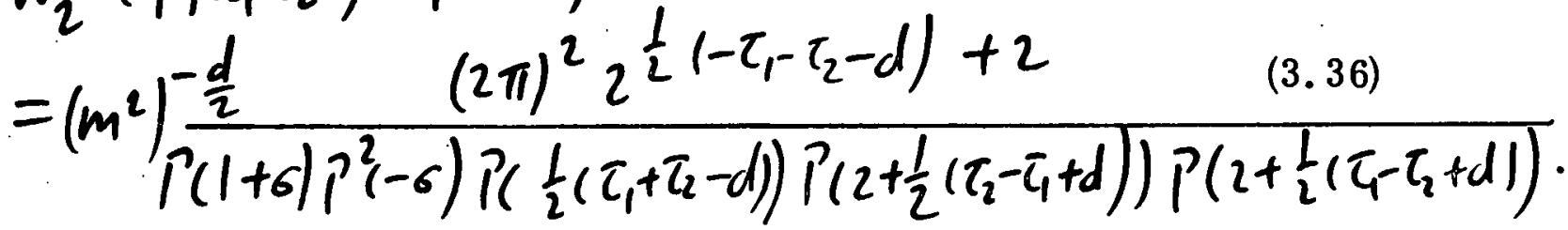

$$
\begin{aligned}
& x \int_{0}^{\infty} d \alpha d \beta(\alpha \beta)^{-\sigma-1} \int d^{4} k\left(-k^{2}-i 0\right)^{d}\left(-(\mathbb{P}-k)^{2}-i 0\right)^{\frac{1}{2}\left(\tau_{1}+\tau_{2}-d\right)-2},
\end{aligned}
$$
carried out in a standard way; the $\alpha$-parametrization, Eq. (3.15) and subsequent rescalings, etc. We obtain:

$$
\begin{aligned}
& W_{2}^{\prime}=\frac{-i\left(m^{2}\right)^{-\frac{d}{2}}(2 \pi)^{4} 2^{\frac{1}{2}}\left(-\tau_{1}-\tau_{2}-d\right) P\left(\frac{1}{2}\left(-\tau_{1}-\tau_{2}-d\right)\right)}{P(1+\sigma) P^{2}(-\sigma) P\left(2+\frac{1}{2}\left(\tau_{2}-\tau_{1}+d\right)\right) P\left(2+\frac{1}{2}\left(\tau_{1}-\tau_{2}+d\right)\right)} \\
& x \frac{P(2+d)}{P(-d) P\left(2+\frac{1}{2}\left(d+\tau_{1}+\tau_{2}\right)\right.} \int_{0}^{\infty} d \alpha d \beta(\alpha \beta)^{-\sigma-1}\left(-P_{-i 0}^{2}\right)^{\frac{1}{2}\left(\tau_{1}+\tau_{2}+d\right)} .
\end{aligned}
$$


$-51-$

The remaining integral is now recognized as the one already given in Eq. (3.35) with $\tau$ replaced by $1 / 2\left(\tau_{1}+\tau_{2}+d\right)$. Therefore it is readily evaluated to give the following complete momentum space representation of $\mathrm{w}_{2}^{\prime}$ :

$$
W_{2}^{\prime}=\left(m^{2}\right)^{-\frac{d}{2}} A\left(\tau_{1} \tau_{2} d\right) W_{2}\left(P, x_{1} x_{2} ; \frac{1}{2}\left(\tau_{1}+\tau_{2}+d\right), \sigma\right)
$$

$$
A\left(\tau_{1} \tau_{2} d\right)=\frac{(2 \pi)^{2} P(2+d) P\left(-\frac{1}{2}\left(\tau_{1}+\tau_{2}+d\right)\right)}{P(-d) P\left(2+\frac{1}{2}\left(\tau_{2}-\tau_{1}+d\right)\right) P\left(2+\frac{1}{2}\left(\tau_{2}+\tau_{1}+d\right)\right)}
$$
and $W_{2}\left(P_{1} x_{1} x_{2} ; \frac{1}{2}\left(\tau_{1}+\tau_{2}+d\right), 6\right)$ is given by Eq. (3.32) replacing $c$ by $1 / 2\left(\tau_{1}+\tau_{2}+d\right)$.

It is worthwhile to remark at this point that the canonical dimersionality of the symmetry breaking field $\mathcal{X}$ is not allowed in this expression since the coefficient A blows up when $d=\mathbf{- 2}$.

3.4) Partial Wave Amplitudes

We now have an explicit expression for the inverse propagator, $G^{-1}$, in both coordinate and momentum space to first order in the tadpole expansion:

$$
\begin{aligned}
& G^{-1}\left(X_{1} x_{1} X_{2} x_{2} ; \tau_{1} \tau_{2} \sigma\right) \\
& =-\delta\left(\tau_{2}-\tau_{1}\right) W_{2}\left(X_{1} x_{1} X_{2} x_{2} ; \tau_{1} \sigma\right)-\left(m^{2}\right)^{-\frac{d}{2}} \int d^{4} X_{3} F\left(X_{1} x_{1} X_{2} x_{2} ; X_{3}\right), \\
& G^{-1}\left(P_{1} x_{1} x_{2} ; \tau_{1} \tau_{2} \sigma\right) \\
& =-\delta\left(\tau_{2}-\tau_{1}\right) W_{2}\left(P, x_{1} x_{2} ; \tau_{1} \sigma\right) . \\
& -\left(m^{2}\right)-\frac{d}{2} A\left(\tau_{1} \tau_{2} d\right) W_{2}\left(P, x_{1} x_{2} ; \frac{L}{2}\left(\tau_{1}+\tau_{2}+d\right), \sigma\right)
\end{aligned}
$$


$-52-$

We are now going to make partial wave expansion of $G^{-1}$ in the rest frame of the momenta $P$, we write:

$$
G^{\dagger}\left(p, x_{1} x_{2} ; \tau_{1} \tau_{2} d\right)=\sum(2 \ell+1) G_{\ell}^{-1}\left(p ; \tau_{1} \tau_{2} d\right) P_{l}(\cos \theta),
$$

where $P_{\ell}(\cos \theta)$ is a Legendre polynomial. First, we will consides $W_{2}$ in detail. The angular dependent part of $W_{2}$ may be written as follows:

$$
f(\theta ; \tau)=\left(\sin ^{2} \frac{\theta}{2}\right)^{2 \sigma-\tau} p_{\tau-\sigma}^{(0,20-\tau)}(-\cos \theta)
$$

where we have used the relation $k \cdot 1=|\underline{k}||l| l \mid(1-\cos \theta)$ which is valid in the rest frame of $P$. The partial wave amplitudes, $f(\ell)$, defined similarly can be calculated, if $\tau-\sigma$ is integer and $\operatorname{Le}(26-\tau)>-1$, using the formula:

$$
\text { 45) }
$$

$$
\begin{aligned}
& \frac{1}{2} \int_{-1}^{1}\left(\sin ^{2} \frac{\theta}{2}\right)^{\alpha} P_{m}^{(\alpha, 0)}(\cos \theta) P_{l}(\cos \theta) d \cos \theta \\
& =(-1)^{l+m} \frac{P(\alpha+m+1) P(\alpha+1) P(l+m+1)}{P(m+1) P(\alpha-l+m+1) P(\alpha+l+m+2) \Gamma(l-m+1)}
\end{aligned}
$$

We find: $\quad($ for $\ell, m=$ integer, $\operatorname{Re} \alpha>-1)$.

$$
\begin{aligned}
f(l ; \tau \sigma) & =\frac{1}{2} \int_{-1}^{1} f(\theta ; \tau \sigma) P_{l}(\cos \theta) d \cos \theta, \\
& =(-1) \frac{P(\sigma+1) P(\sigma-v+1) P(l+v+1)}{P(v+1) P(\sigma-l+1) P(\sigma+l+2) \Gamma(l-v+1)},
\end{aligned}
$$


where we defined $V=\tau-\sigma$, and also used the relation:

$$
p_{m}^{(\alpha, \beta)}(x)=(-1)^{m} p_{m}^{(\beta, \alpha)}(-x)
$$

Since Eq. (3.43) is defined only in the region where $\nu, l$ are integer and $R_{e} \sigma>U-1$, we need to make the analytic continuation in order to define the amplitudes, $f(\ell)$ for other values of the variables. At this point let us note that $f(l)$ is a meromorphic function of the complex variables $\ell$ and $\nu$. Now the crucial 46) question is whether we can apply the Carlson's theorem in each variable $\ell, \nu$ separately. If it is possible, then the Hartog's 47)

theorem will tell us that we have a unique analytic continuation of the function $f(\ell)$ in both $\ell$ and $V$. Since the above mentioned two theorems are of fundamental importance, we wish to quote them here.

Carlson's Theorem

Let $f(Z)$ be an analytic function in $\operatorname{Re} z \geqslant a$ and if $f(Z)=$ $O\left(e^{\lambda|z|}\right)$ with $\lambda<\pi$, when $|z| \rightarrow \infty, \operatorname{Re} z \geqslant a$. Then the function $f(Z)$ is uniquely determined by its values at the integral values of $\mathbf{Z}$.

Hartog's Theorem

When a function $f\left(z, z^{\prime}\right)$, for values of $|z| \leqslant r$ and of $\left|z^{\prime}\right| \leqslant r^{\prime}$ is a regular function of $\mathbf{Z}$ everywhere within the assigned $\mathbf{z}$-circle 
for every value of $Z^{\prime}$ within its assigned circle and also is a regular function of $\mathrm{Z}^{\prime}$ everywhere within the assigned $\mathrm{Z}^{\prime}$-circle for every value of $Z$ within its assigned circle, it is a regular function of $\mathrm{Z}$ and $\mathrm{Z}^{\prime}$ everywhere within the indicated field of $\mathrm{Z}, \mathrm{Z}^{\prime}$ variation.

Equipped with the above two theorems, let us now examine the asymptotic behaviour of $f(q)$ This will be done by using the standard asymptotic expansion for the $\Gamma$-function:

$P(2)=e^{-2} 2^{2-\frac{1}{2}}(2 \pi)^{\frac{1}{2}}\left(1+\frac{2}{12}^{-1}+\cdots\right)$

for $|\arg z|<\pi$.

It is easily seen that Eq. (3.43) is not suitable for the analytic continuation since,

$$
\frac{P(\ell-v+1)}{P(\sigma-\ell+1) P(\sigma+\ell+2) P(\ell-v+1)} \longrightarrow e^{\pi|\ell|}
$$

and also the factor $(-1)^{\ell}$ causes trouble. However, this can be avoided by defining signatured amplitudes when they are necessary. Therefore we have to recast Eq. (3.43) into a form which is suitable for the continuation. This can be done by using the relation:

$$
(-1)^{\ell+1} P(\sigma-l+1) P(l-\sigma)=\frac{\pi}{\sin \pi \sigma} \text {, }
$$

for $\ell=$ integer.

We now have: 
$-55-$

$$
f(l ; v \sigma)=-\frac{\sin \pi \sigma}{\pi} \frac{P(\sigma+1) P(\sigma-v+1) P(\ell+v+1) P(\ell-\sigma)}{P(v+1) P(\sigma+\ell+2) P(\ell-v+1)}
$$

We now investigate the asymptotic behaviour of Eq. (3.47) in each variable $\ell$ and $\dot{V}$ separately.

a) $|\ell| \rightarrow \infty, L$

$$
f(l ; v \sigma) \rightarrow-\frac{\sin \pi \sigma}{\pi} \frac{p(\sigma+1)}{p(v+1)}|l|^{-26-2 v-2}
$$

This is polynomially bounded, hence the Carlson's theorem is applicable. We have a unique analytic continuation in $\ell$.

b) $|\nu| \rightarrow \infty, \ell$

$$
\left.f(l ; v \sigma) \longrightarrow-\frac{\sin \pi \sigma}{\pi} \frac{P(\sigma+1) P(l-\sigma)}{P(\sigma+\ell+2)} \mid \nu\right)^{\sigma+2}
$$

The Carlson's theorem is again applicable and we have a unique continuation in $U$ as a consequence.

Combining a) and b) we are guaranteed to have a unique analytic continuation in both variables $\ell, \nu$ according to the Hartog's theorem. This completes our assertions about the partial wave amplitude of $\mathrm{W}_{2}$.

Next we go over to the correction term $\mathrm{w}_{2}{ }^{1}$. In a similar way as in $\mathrm{W}_{2}$, the angular dependent part is seen to be:

$$
\left.f^{\prime}\left(v ; \tau_{1} \tau_{2} \sigma\right)=\left(\sin ^{2} \frac{\theta}{2}\right)^{2 \sigma-\frac{l}{2}\left(\tau_{1}+\tau_{2}+d\right)}\right)_{\frac{1}{2}\left(\tau_{1}+\tau_{2}+d\right)-\sigma}^{\left(0,2 \sigma-\frac{1}{2}\left(\tau_{1}+\tau_{2}+d\right)\right)} \begin{aligned}
& (-\infty, 0))^{(3.50)} \\
& .
\end{aligned}
$$


$-56-$

Evidently the partial wave amplitudes $f^{\prime}(\ell)$ is now given by Eq.

(3.47) with $\nu$ replaced by $\nu^{\prime}$, where $\nu^{\prime}$ is defined by $U^{\prime}=\frac{1}{2}\left(\tau_{1}+\tau_{2}+d\right)-\sigma$. On applying similar steps which we carried out for $f(\ell)$ we can easily see that a unique continuation in $\ell$ and $\nu^{\prime}$ exists for the amplitudes $f^{\prime}\left(\ell ; v^{\prime}\right)$ as well. Summarizing what we obtained so far, we may write:

$$
\begin{aligned}
& G_{l}^{-1}\left(p ; \tau_{1} \tau_{2} \sigma\right)=-\delta\left(\tau_{1}-\tau_{2}\right) w_{2}\left(l, p ; \tau_{1} \sigma\right)- \\
& -\left(m^{2}\right)^{-\frac{d}{2}} A\left(\tau_{1} \tau_{2} d\right) w_{2}\left(l, p ; \frac{1}{2}\left(\tau_{1}+\tau_{2}+d\right) \sigma\right),
\end{aligned}
$$

where $W_{2}(\ell, p ; \tau, \sigma)$ is given by

$$
W_{2}=\frac{2^{\sigma}(2 \pi)^{2}}{P\left(2+\tau_{1}\right) P\left(-\tau_{1}\right)^{1}} P\left(\sigma-\tau_{1}\right)\left(-\frac{p^{2}}{2}\right)^{\tau_{1}-\sigma}(|k||l|)^{\sigma} f\left(l ; \tau_{1} \sigma\right)^{(3.52)}
$$

One notices that the definition of $\cos v$ is Lorentz invariant, and further that the vectors

$$
\begin{aligned}
& k_{\mu}-\frac{1}{p^{2}}(k \cdot p) p_{\mu}, \\
& l_{\mu}-\frac{L}{p^{2}}(k \cdot l) p_{\mu},
\end{aligned}
$$

become $\left(0, k_{2}\right)$ and $(0, \ell)$ respectively in the rest frame of $P$, we may rewrite the partial wave amplitudes in a manifestly covariant form:

$$
\begin{aligned}
& w_{2}(l, P ; \tau \sigma) \\
& =\frac{2^{\sigma}(2 \pi)^{2}}{P(2+\tau) P(-\tau) i} P(\sigma-\tau)\left(-\frac{p^{2}}{2}\right)^{\tau-\sigma}\left(\frac{(p \cdot k)(p \cdot l)}{p^{2}}\right)^{\sigma} f(l ; \tau \sigma) .
\end{aligned}
$$


For $p^{2}<0$ (space-like momenta), the series, Eq. (3.40), has to be rewritten as a Sommerfeld-Watson integral:

$$
G^{-1}\left(p x_{1} x_{2} ; \tau_{1} \tau_{2} \sigma\right)=\frac{i^{\prime}}{2} \int_{C} d l \frac{P_{l}(-\cos \theta)}{\sin ^{\prime} \pi l}(2 l+1) G_{l}^{-1}\left(p ; \tau_{1} \tau_{2}\right)(3.54)
$$

where the contour C may be chosen, for example

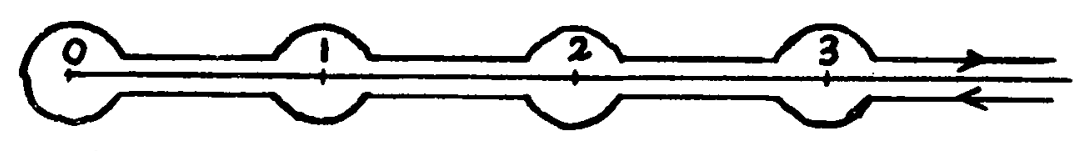

Fig. 4

It is interesting to observe that $G_{\ell}^{-1}$ is singularity free through the entire right-half plane when the representations are on the principal series, therefore we can open up the contour without picking up any singularities unless we cross the line $\operatorname{Re} l=-1$. 3.5) Regge Trajectories; the Hadron Spectrum

The singular surfaces of the Green's function (or equivalently, regular null surfaces of $\mathrm{G}^{-1}$ ) :are investigated explicitly up to first order in the tadpole expansion. By doing this we are able to obtain the spectrum of the physical particle states which is qualitatively very encouraging to our symmetry breaking scheme. Before proceeding, it is to be remarked that a perturbation series expansion of the Green's function in powers of the symmetry breaking parameter necessarily breaks down at its singular surfaces; on the other 
$-58-$

hand it is believed that the series expansion makes sense for $\mathrm{G}^{-1}$. This is essentially the reason why we are studying $G^{-1}$ instead of $G$. In what follows, we study the regular null surfaces of the inverse propagator by the Brillouin-Wigner variational techniques.

As we have seen, the symmetry breaking term mixes the conformal weights; $\tau$ is no longer a good quantum number of the perturbed system. Hence, we have to introduce new effective fields which diagonalize the quadratic part of the effective action. It follows from the orthogonality property of the partial wave expansion that each partial wave may be considered separately in the following calculation. To begin with, we make the following ansate for the perturbed effective field:

$$
|\alpha\rangle=z^{-\frac{1}{2}}\left(|\tau\rangle+\left(m^{2}\right)^{-\frac{d}{2}} p \rho d \tau^{\prime} a\left(\tau^{\prime}\right)\left|\tau^{\prime}\right\rangle\right),
$$

where $\mathcal{P} \int d \tau^{\prime}$ implies that the integration is extended over the principal series, ide., $\operatorname{Re} \tau^{\prime}=-1$, and $\mathrm{z}$ is a normalization constant which is determined by the condition $\langle\alpha \mid \alpha\rangle=1$ :

$$
Z=1+\left(m^{2}\right)^{-\frac{d}{2}} p \int d \tau^{\prime} a\left(\tau^{\prime}\right) \overline{a\left(\tau^{\prime}\right)} \text {. }
$$

The perturbed eigenvalue $\alpha(\tau)$ is chosen such that:

$\lim _{m^{2} \rightarrow 0} \alpha(\tau)=\tau$ $m^{2} \rightarrow 0$ 
$-59-$

We also denote that the eigenvalue of the unperturbed inverse propagator, $W_{2}(\ell, p)$, in the $\tau$-representation by $h(\tau)$. The coefficients $a\left(\tau^{\prime}\right)$ in Eq. (3.55) are determined by imposing the condition that the perturbed effective fields extremize the quadratic part of the perturbed effective action (see Sec. 3.1).

$$
\frac{\delta \omega}{\delta \bar{a}\left(\tau^{\prime}\right)}=\frac{\delta}{\delta \bar{a}\left(\tau^{\prime}\right)}\left\langle\alpha\left|G_{l}^{-1}\right| \alpha\right\rangle=0 \text {, }
$$

where $\omega$ is the eigenvalue of $G_{l}^{-1}$ in the $\alpha$-representstimon.

From the Eqs. (3.55), (3.56), (3.58) we obtain for $a\left(\tau^{\prime}\right)$ :

$$
a\left(\tau^{\prime}\right)=-\frac{\left\langle\tau^{\prime}\left|w_{2}^{\prime}(l, p)\right| \tau\right\rangle}{\omega-\beta\left(\tau^{\prime}\right)},
$$

where $\omega$ should satisfy:

$\omega=\beta(\tau)+\left(m^{2}\right)^{-d} p \int d \tau^{\prime} \frac{\left\langle\tau\left|w_{2}^{\prime}\right| \tau^{\prime}\right\rangle\left\langle\tau^{\prime}\left|w_{2}^{\prime}\right| \tau\right\rangle}{\omega-\beta\left(\tau^{\prime}\right)}$

and $\beta(\tau)$ is given by:

$$
\beta(\tau)=h(\tau)+\left(m^{2}\right)^{-\frac{d}{2}}\left\langle\tau\left|w_{2}^{\prime}(\ell, p)\right| \tau\right\rangle \text {. }
$$

Further, let us define the function $\mathrm{H}(\boldsymbol{z})$ by:

$$
H(z)=z-\beta(\tau)-\left(m^{2}\right)^{-d} p \int d \tau^{\prime} \frac{\left\langle\tau\left|w_{2}^{\prime}\right| \tau^{\prime}\right\rangle\left\langle\tau^{\prime}\left|w_{2}^{\prime}\right| \tau\right\rangle}{z-\beta\left(\tau^{\prime}\right)}
$$

Evidently we recover Eq. (3.60) by setting $\mathrm{H}(\boldsymbol{\omega})=0$. It can be also shown that the normalization constant $\mathrm{Z}$ is given by the equation,

$$
Z=\left(\frac{\partial H}{\partial z}\right)_{z=\omega} \text {. }
$$


These equations give the general solution to the symmetry breaking problem as defined in the present chapter. Now the singular surfaces of the Green's function are given by the vanishing eigenvalues, $w=0$. From Eq. (3.60), we see easily that these are determined by the equation:

$\beta(\tau)=\left(m^{2}\right)^{-\frac{d}{2}} p \int d \tau^{\prime} \frac{\left\langle\tau\left|w_{2}^{\prime}\right| \tau^{\prime}\right\rangle\left\langle\tau^{\prime}\left|w_{2}^{\prime}\right| \tau\right\rangle}{\beta\left(\tau^{\prime}\right)}$.

It is to be pointed out that the problem treated here closely resembles that of certain non-relativistic many-body systems which exhibit spontaneous symmetry breaking. The omission of terms higher than quadratic ones in the action corresponds to the free quasi-particle approximation in the non-relativistic many-body problem. We now examine Eq. (3.64) to lowest order in the symmetry breaking parameter. Clearly, the results should be treated with some caution. Nevertheless, it is reasonable to expect that the leading singularities of the Green's function will be reproduced up to small corrections. In this order, we have:

$$
\beta(\tau)=0
$$

This is easily seen to be given by:

$$
\begin{aligned}
\beta(\tau) & =W_{2}(l, p j \tau \sigma)\left[1+\left(\frac{-p^{2}}{4 m^{2}}\right)^{\frac{d}{2}} B \frac{\Gamma\left(l+v^{\prime}+1\right) P(l-v+1)}{P(l+v+1) P\left(l-v^{\prime}+1\right)}(3.66)\right. \\
& =0,
\end{aligned}
$$

where

$$
B=\frac{P\left(\sigma-v^{\prime}+1\right) P(v+1)}{\Gamma(\sigma-v+1) P\left(v^{\prime}+1\right)} A(\tau \tau d) \text {. }
$$


The physical particle states are determined by the roots of the square bracket in Eq. (3.66). In the SU $(2,2)$ limit all discrete levels of the system must have zero mass, hence we look for such solutions of the first order formula, Eq. (3.66), which are close to massless states. The factor $\left(-\frac{p^{2}}{4 m^{2}}\right)^{\frac{d}{2}}$ blows up when $p^{2} \rightarrow 0$, hence the solutions must be close to the poles of the $\Gamma$-functions in the denominator. We now approximate $\Gamma(\mathrm{Z})$ by using the relation:

$$
\lim _{z \rightarrow-n}(z+n) \Gamma(z)=\frac{(-1)^{n}}{n !} .
$$

The other factors which depend on $\ell$ are evaluated at the position of the poles as usual. We may also write $d=-2$ in every factor where this does not give rise to infinities if the deviation from the canonical dimensionality is really small. This is indeed the case as we will see shortly. In this way we arrive at the following approximate equation for the leading singular surfaces which come from the poles of $\Gamma(\ell+V+1)$ :

$S_{n}(s, l, v)=\frac{\pi \beta(l+v+1+n)}{(n+1)(n+2 v) \sin \pi\left(-\frac{d}{2}\right)}+\left(\frac{-s}{4 m^{2}}\right)^{-\frac{d}{2}}=0$

where we put $s=p^{2}$.

This gives rise to a series of Regge trajectories, $l=\alpha_{n}(s)$, with intercept at $-V-\mid-n$. One, also, must notice that at fixed $\ell$ Eq. (3.68) gives an almost linear relationship between 
masses and scale dimensions - thereby recovering the dilatational trajectories which we mentioned before. The Regge trajectories, $\ell=\alpha_{n}(S)$, have several interesting qualitative features. The fact that the $S$-dependence occurs in a form $\sim(-S)^{1-\delta}$ (we let $d=-2+2 \delta$ ) predicts the total width over mass ratios should be a universal constant. This is because the total width of a resonance of mass $M$ lying on such a trajectory is given by:

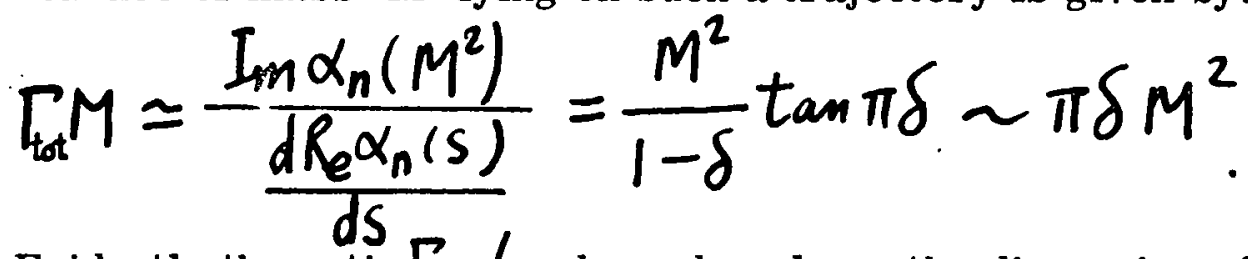

Evidently the ratio $\Gamma_{t o t} / M$ depends only on the dimension of the symmetry breaking term and hence it should be universal. In order to test this prediction, we fitted the total widths of all the well established baryon resonances 4 ) a curve:

$$
\Gamma_{\text {tot }} M=\pi \delta\left(M^{2}-M_{0}^{2}\right) \text {, }
$$

where $M_{0}$ was introduced in order to take threshhold effects roughly into account. This is shown in Fig. 5. Indeed the data are approximately consistent with Eq. (3.70) with a universal value of $\delta \sim 0.04$. A similar pattern is found in the case of meson resonances. However, experimental uncertainties prevent one from drawing any firm conclusions at this time. It is also interesting to notice that the slope of the Regge trajectories tend to infinity in the SU $(2,2)$ limit, i.e., $m^{2} \rightarrow 0$. Hence all the excited states collapse 


\section{$\Gamma M\left(G_{e V}{ }^{2}\right)$}

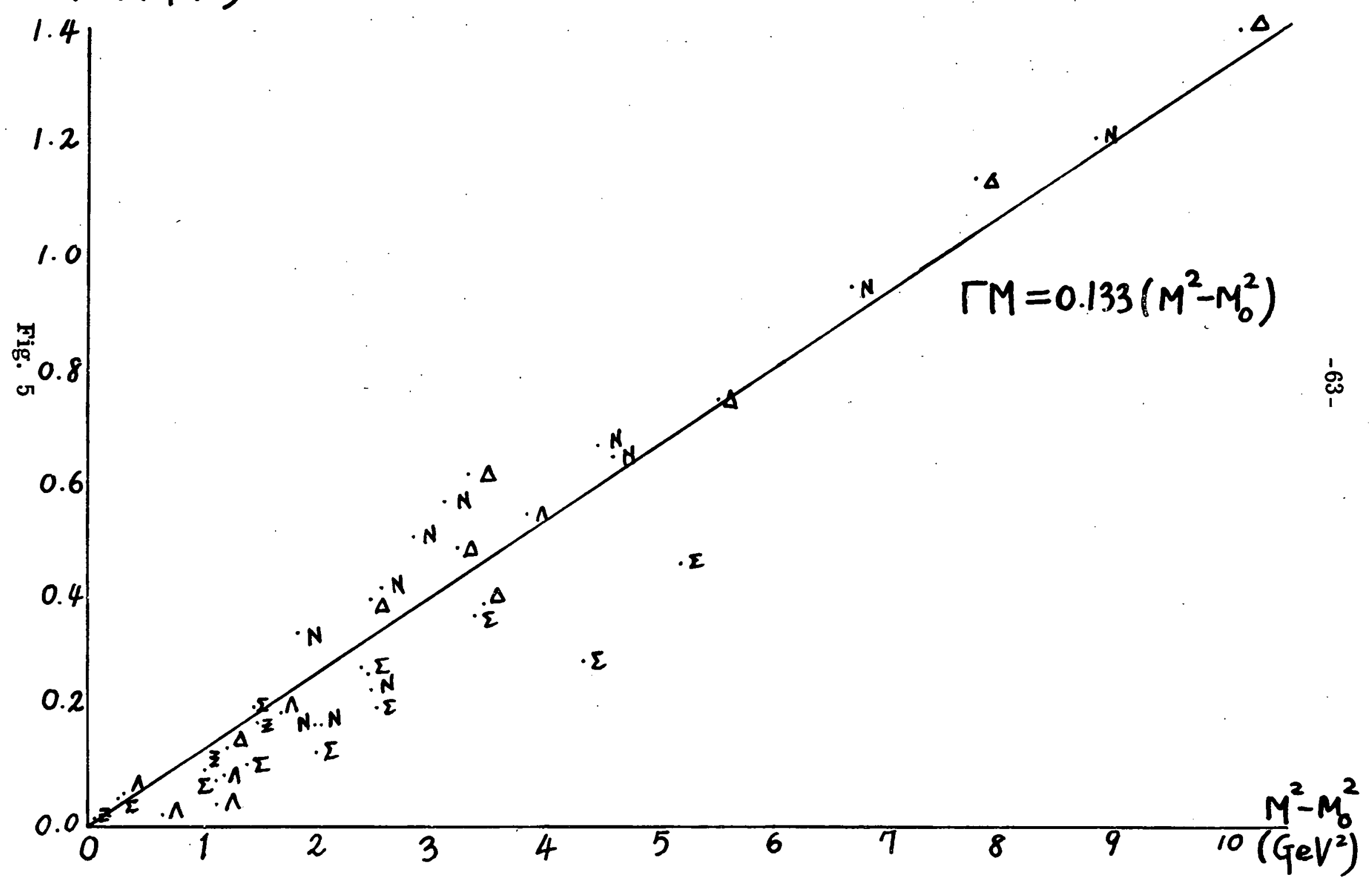


into an infinitely degenerate massless object. Incidentally this is what we expected when we assigned the $\mathrm{SU}(2,2)$ covariant field $\varphi^{\infty}$ to the hadrons.

As we have emphasized before, the results of the first order calculation cannot be trusted seriously in a quantitative sense. In particular, the positions of the daughter trajectories for $S \neq 0$ may be substantially affected by higher order terms in the tadpole expansion. (For more discussion on this point, see Sec. 3.6.) It should be also noted that the threshhold of the Regge trajectories lie at $\mathbf{S}=0$. This is because the tadpole expansion is not unitary in each order separately. Finally we notice that the trajectories, $S_{n}^{\prime}(S, \ell, v)$, which arise from the poles of $\Gamma\left(\ell-v^{\prime}+1\right)$ are low lying so that they contribute to non-leading singularities. In fact:

$S_{n}^{\prime}=\frac{\pi B\left(1+l-\nu^{\prime}+n\right)}{(n+1)(n+2-2 v) \sin \pi\left(-\frac{d}{2}\right)}+\left(\frac{-S}{4 m^{2}}\right)^{-\frac{d}{2}}=0$

Hence the leading trajectory of $S_{n}^{\prime}$ intercepts the $l$ axis if we assume $d \sim-2, \nu \sim-1$ (i.e. around canonical values). This is around the position where the third daughter trajectory of $S_{n}$ intercepts the $\ell$ axis. 


\section{6) Remarks}

First, it should be noted that the calculations which we have performed are also equally applicable to fermions. This can be seen from the fact that the fermion propagator in momentum space is equal to boson propagators, multiplied only by a factor $\gamma^{\mu} p_{\mu}$ in the frame work of orbital excitation model which we have chosen as a possible theory. Therefore the structure of singularities which depends on the orbital part should be quite similar. Even though such a complete decoupling between a spin and orbital part may not be the case in general, we believe the singularity structures should not be qualitatively much different.

Secondly, we would like to speculate about the behaviour of Regge trajectories, Eqs. (3.68), (3.71). As we already noted, the first order calculations cannot be taken too seriously in a quantitative sense, however, keeping this caition in mind, some speculations may be helpful in understanding our results. As they stand, the Regge trajectories rise almost linearly with mass square; this is an extrapolation since, strictly speaking our results Eqs. (3.68), (3. 71), are valid only in the neighbourhood of small $\boldsymbol{S}$. In order to see the behaviour of trajectories at high $\boldsymbol{S}$, we have investigated Eq. (3.66). Since the factor $\left(-\frac{5}{4 m^{2}}\right)^{\frac{d}{2}}$ approaches zero for $|s| \rightarrow \infty$, the solutions must be close to the poles of the $\Gamma$ - 
functions in the numerator. In this way we find two classes of

$$
\begin{aligned}
& \text { decreasing trajectories with } S: \\
& l=-1+V-n+\frac{B \sin \pi\left(-\frac{d}{2}\right)}{\pi n(n+1-2 V)}\left(\frac{-S}{4 m^{2}}\right)^{\frac{d}{2}}, \\
& l=-1-V^{\prime}-n+\frac{B \sin \pi\left(-\frac{d}{2}\right)}{\pi n(n-1+2 V)}\left(\frac{-5}{4 m^{2}}\right)^{\frac{d}{2}} .
\end{aligned}
$$

Hence it is quite tempting to conjecture - combining Eqs. (3.68), (3.71), (3.72) - that the trajectories will rise almost linearly to some point and approach constant values asymptotically.

If turning points are sufficiently high-lying to give many resonances, we would get a very complicated resonance spectrum $w$ ith an increasing degeneracy of the states.

Finally we would also like to mention what are the possible effects of the higher order $(\boldsymbol{n} \geqslant 2)$ correction terms in the tadpole series. One cannot be too precise on this point since the higher order terms contain arbitrary functions as coefficients. As is easily seen, they are homogeneous functions of $p^{2}$; the general $n$-th order term of the tadpole expansion is shown to be proportional to:

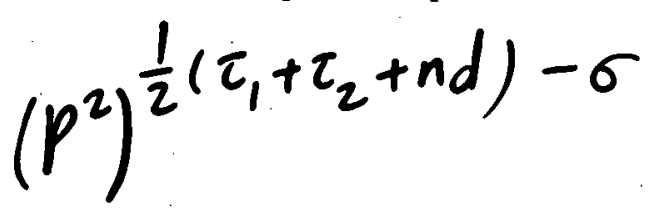

by a simple power counting argument. Our preliminary estimates suggest that the $n$-th order correction term to the partial wave amplitudes contains a factor typically like $\Gamma\left(l-v-\frac{n d}{2}-1\right)$ which would give a leading trajectory with an intercept at $-1+v+\frac{n d}{2}$ and subsequent its daughters. 
Chapter 4

Hadronic Scaling

\section{1) Introduction}

One of the most prominent features in high energy hadron collisions $^{49)}$ - at center of mass energies of a few GeV or more is that there is a stringent limit on the transverse momenta $q_{T}$ of all outgoing particles. They are typically of the order 0.3 to $0.4 \mathrm{GeV} / \mathrm{c}$ and are largely independent of the incident energy and the type of reactions studied. Therefore it is in general a good approximation at accelerator energies to describe the dependence in $\mathrm{q}_{\mathrm{T}}$ by a sharply decreasing exponential distribution. It is also known that most of the produced particles in such collision processes are pions. (About $90 \%$ of the products at $20 \mathrm{GeV}$.) However, there is reason to believe that the situation may be quite different in the region of large transverse momenta. (Say, $\left.\mathrm{q}_{\mathrm{T}}>3 \mathrm{GeV} / \mathrm{c}\right)$. In fact, recent experimenters at CERN - ISR ${ }^{50)}$ have discovered that the distribution in $\mathrm{q}_{\mathrm{T}}$ becomes wider, showing a clear deviation from the exponential dependence at small $\mathrm{q}_{\mathrm{T}^{\cdot}}$ It is also expected that one may see more strange particle production than what one would expect from an extrapolation of the data at low $\mathrm{q}_{\mathrm{T}^{*}}$ This is of extreme importance since it may lead us to a new understanding of hadronic reactions. 
$\Lambda$ quite remarkable property of high energy hadronic reactions is the appearance of phenomena, called "scaling". Such phenomena have been known for some time and have been extensively studied for the deep-inelastic lepton-hadron scattering processes. 6,7 ). However, scaling in purely hadronic reactions, ${ }^{50)}$ such as $p+p \rightarrow \pi+$ anything, has been observed only recently. (In order to avoid a possible misunderstanding, we wish to emphasize that the scaling phenomena discussed here are different from the "Feynman scaling" 51 ) observed at low transverse momenta. As is well known, Feynman's scaling hypothesis states that the inclusive single particle distribution depends on the observed longitudinal momentum only through the variable $x=\frac{\mathbf{8}_{11}}{\sqrt{5}}$. However, the magnitude of the transverse momentum remains fixed and is about $\sim 0.4 \mathrm{GeV} / \mathrm{c}$. The scaling properties discussed here refer to the kinematic region where all the independent kinematic invariants are large, - typically $2 \mathrm{GeV}$ or larger - and thus they are analogues of the "Bjorken scaling" observed in inelastic lepton scattering.) It is well known that the scaling phenomena observed in the lepton-induced reactions may be explained by assuming that hadrons are composite and their constituents known as "partons" 52) - behave as if they were point-like particles in the infinite momentum frame of the hadrons. In 
describing hadronic reactions at large transverse momenta, it is quite tempting to apply parton concept since we are essentially probing the short distance structure of the hadrons. In fact, various parton models ${ }^{53)}$ have been quite successful in explaining "hadronic scaling". However, it is quite conceivable that scaling phenomena reflect more general aspects of the dynamics of hadrons than is suggested by the specific models.

In this chapter we propose that the hypothesis of asymptotic conformal invariance, together with some additional, rather general, assumptions leads to an understanding of at least the main qualitative features of the data on hadron induced inclusive reactions in this, "scaling region".

A general framework to study the inclusive reactions,

$$
\mathrm{P}_{1}+\mathrm{P}_{2} \longrightarrow \mathrm{q}+\text { anything, }
$$

has been introduced by Mueller. ${ }^{54)}$ He has observed that the cross section of an inclusive reaction, $q^{\circ} \frac{d^{3} \sigma}{d q^{3}}$, can be regarded as a discontinuity in a six-point forward scattering amplitude. This is a generalization of the relation - known as the "optical theorem" between the total cross section and the absorptive part of a forward scattering four-point function. So we have at high energies:

$$
q^{0} \frac{d^{3} \sigma}{d q^{3}} \sim \frac{1}{s} A
$$

where $\mathbf{A}$ denotes the absorptive part just mentioned. 
In the following sections we study a six-point conformal amplitude in its most general form except spins. (We have neglected all the spins for simplicity of calculations). A scaling law follows immediately in our scheme. However, a specific form of the scaling function can only be obtained by making certain assumptions on the form of the amplitude. To be more specific we assume that an arbitrary generalized reduced matrix element is a completely factorizable function of the independent channel variables, $c_{i}$. This is a mathematical interpretation of the physical assumption that the dynamics responsible cor the process under consideration is a very complicated one and hence dynamical correlation effects are cancelled out to a good approximation; we call this an independent correlation model.

It is found that we can fit the available experimental data on $\mathrm{pp} \rightarrow \pi^{\circ} \mathrm{X}$ excellently with the scaling function so obtained. We also present the predicted production rates for the reactions $\mathrm{pp} \rightarrow \mathrm{KX}$ and $p p \rightarrow \eta x$.

\subsection{Six-point Conformal Amplitude}

In what follows, calculations will be done under two simplifying as sumptions;

a) We neglect all spins, so we work with a Symanzik representation instead of the more general DK-representation previously described in Ch. 2 .

b) We work to zeroth order in the symmetry breaking tadpole. However, this is consistent with the first order calculation of the spectrum. We begin by drawing a six-point conformal diagram. 
71

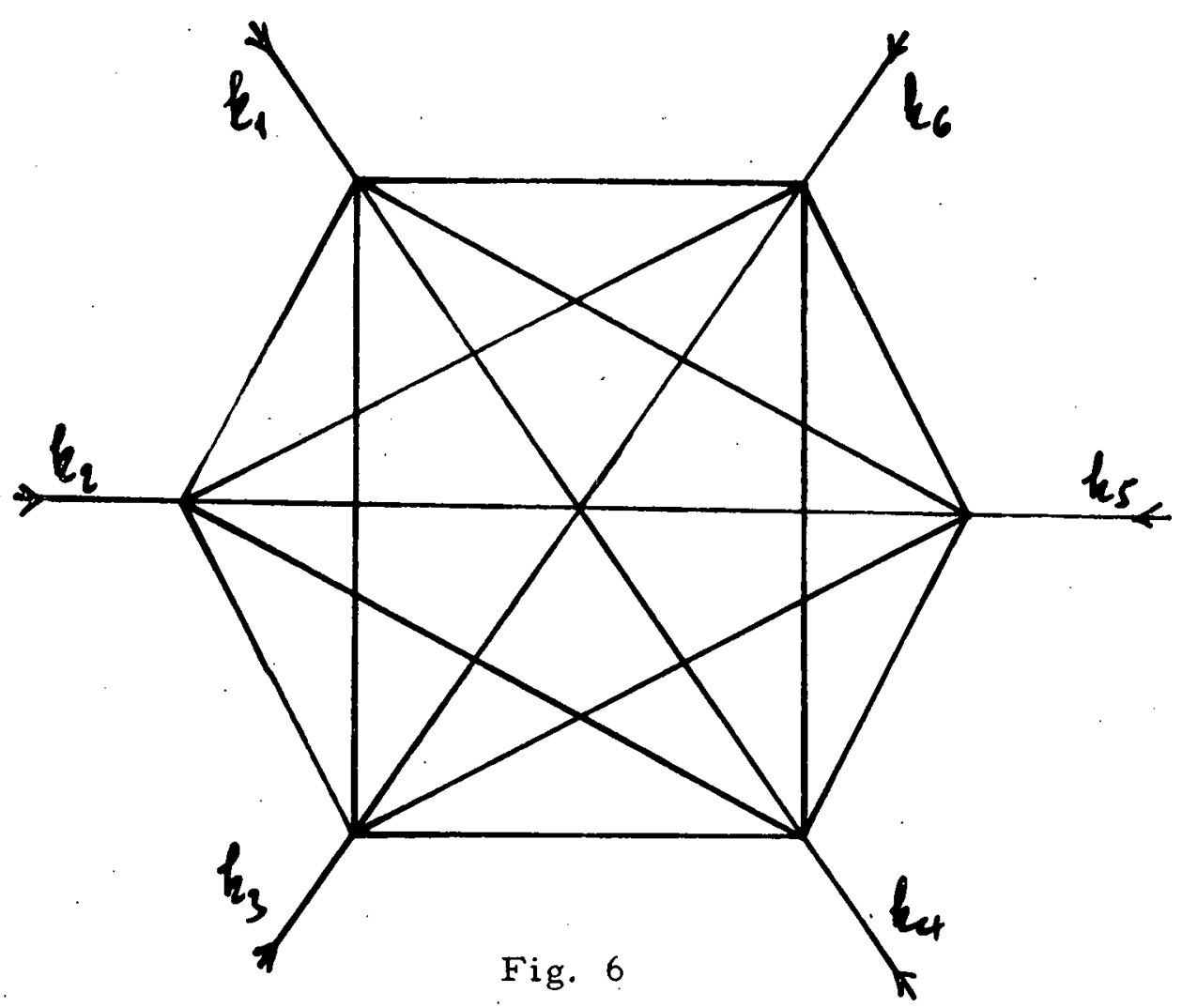

The conformal covariant six-point amplitude, $\mathrm{T}_{6}$, may be written in a straightforward manner by the familiar construction principles:

$$
\begin{aligned}
& T_{6}\left(x_{1}, \cdots, x_{6}\right)=\int \cdots \int \pi d \tau_{j k}\left(x_{j k}{ }^{2} \tau_{j k} p\left(-\tau_{j l}\right) .\right. \\
& \cdot \prod_{j} \delta\left(\sum_{b} \tau_{j k}+\tau_{j}+4\right) G_{0}\left(\tau_{12}, \cdots, \tau_{56}\right),
\end{aligned}
$$

where $G_{0}$ is an arbitrary function of scale dimensions $\tau_{j k}$ and $\boldsymbol{x}_{j k} \mu$ $=\boldsymbol{x}_{j}{ }^{\mu}-\boldsymbol{x}_{\boldsymbol{k}}^{\mu}$ denote spacetime differences. In writing this form of $\mathrm{T}_{6}$, we have absorbed certain constant factors, e. g. $\left(-\frac{1}{2}\right)^{\tau_{j k}}$ into $\mathrm{G}_{0}$. Instead of working directly with (4.2) we find it more convenient to derive an equivalent form which turns out to be more suitable to calculate the Fourier transform and to our subsequent calculations.

Consider an amplitude F: 
72

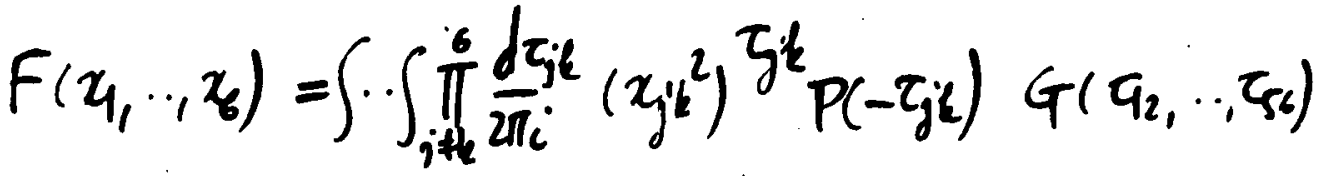

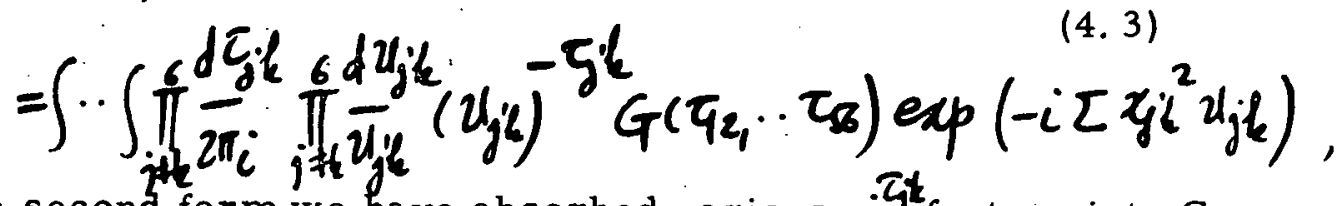

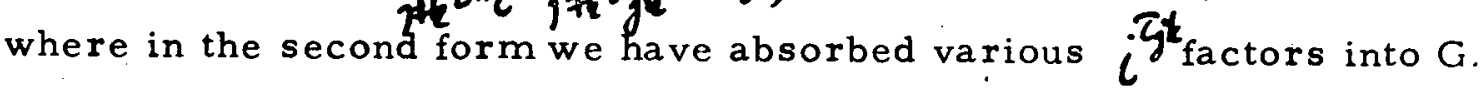

Evidently $F$ does not represent a conformal amplitude unless the internal dimensions satisfy the covariance conditions. However, we can project out conformal portions of the amplitude $F$ by using the Mellon projection technique which reads as follows:

Let $F(x)$ be an arbitrary function which is in general not homogeneous. We can project out a "homogeneous component" of degree $\alpha$ in $\chi$ by

$$
F_{\alpha}(x)=\int_{0}^{\infty} \frac{d \lambda}{\lambda} \lambda^{-\alpha} F(\lambda x)
$$

It is easy to see that $F_{\alpha}(u x)=u^{\alpha} F_{\alpha}(x)$. A necessary generalization to functions of many variables can be done without any difficulty. In view of $E_{i}$. (4.4) and its subsequent generalization we construct an amplitude $\mathrm{F}_{\mathrm{C}}$ from the amplitude $\mathrm{F}$ by multiplying each $z_{j k}^{2}$ by $\mu_{j} \mu_{k}$ and integrating over $\mu_{j}$ for each vertex $j$ with a factor $\mu . j+3$ from 0 to $\infty$.

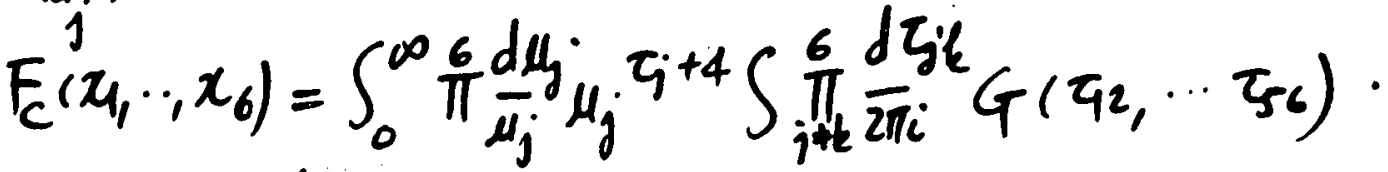

$$
\begin{aligned}
& \cdot \int_{0}^{\infty} \prod_{j+k}^{6} \frac{d w_{j l} k}{z_{j l}} u_{j k}^{-j_{j} k} \exp \left(-i \sum_{j, k}^{6} x_{j l}^{2} u_{j k} u_{j} \mu_{k}\right) \text {. }
\end{aligned}
$$

It is easily checked that the amplitude $F_{C}$ is indeed a conformal amplitude. For example, we see under a special conformal transformation (2.1) 
73

$$
F_{c}\left(x_{1}^{\prime}, \cdot x_{6}^{\prime}\right)=\frac{6}{\pi}\left|1-2 c \cdot x_{j}+c^{2} x_{j}^{2}\right|^{\tau_{j}+4} F_{c}\left(x_{1}, \cdot x_{c}\right)
$$

Let us now introduce new variables, $a_{j}(1 \leq j \leq 6)$ and $c_{j}(1 \leq j \leq q)$ as follows:

$$
\begin{aligned}
& u_{j j+1}=a_{j} a_{j+1} c_{j}, \\
& u_{j j+2}=a_{j} a_{j+2} c_{j} \cdot c_{j+1}, \\
& u_{j j+3}=a_{j} a_{j+3} c_{6+j} .
\end{aligned}
$$

(In these equations the cyclic identification of the vertices, viz.

$\boldsymbol{u}_{j, 6+\boldsymbol{l}}=\boldsymbol{u}_{\boldsymbol{j}} \boldsymbol{k}$ is implied.) we see now that 6 variables, $\boldsymbol{a}_{\boldsymbol{j}}$. may be eliminated by a suitable redefinition of the reduced matrix element G. In fact, introducing new variables $\boldsymbol{M}_{\boldsymbol{j}}$ by

$$
m_{j}=\mu_{j} a_{j}
$$

we obtain a following representation of the amplitude $F_{C}$ :

$$
\begin{aligned}
& E_{c}\left(x_{1}, \cdots, x_{c}\right)=\int_{0}^{\infty} \prod_{j}^{\infty} \frac{6 m_{j}}{m_{j}} m_{j} \tau_{j}+4 . \int_{0}^{\infty} \pi \frac{q d c_{j}}{c_{j}} K\left(c_{1}, \cdot c_{q}\right) . \\
& \quad \exp -i \Sigma\left(x_{j j+1}^{2} m_{j} \cdot m_{j+1} c_{j}+x_{j j+2}^{2} m_{j} m_{j+2} c_{j} c_{j+1} \ldots\right),
\end{aligned}
$$

where

$$
\begin{aligned}
& K\left(c_{1}, \cdot \cdot, c_{q}\right)=\int_{0}^{\infty} \prod \frac{d a_{j}}{a_{j}} a_{j}^{-\tau_{j}-4} G\left(u_{12}, \cdots, u_{s_{6}}\right) \text {, } \\
& G\left(u_{p_{1}}, \cdots, u_{56}\right)=\int \frac{i}{\pi} \frac{d \tau_{j h}}{2 \pi_{i}}\left(u_{j k}\right)^{-\tau_{j k}} G\left(\bar{u}_{2}, \ldots, \tau_{56}\right) \text {. }
\end{aligned}
$$

(It is understood that the arguments of $G\left(u_{12}, \ldots U_{r_{6}}\right)$ are expressed through the new variables, $a_{j}$ and $c_{j}$, by means of $E_{q} .(4.6)$.) In obtaining the representation (4.7) we have used the observation that the volume element is invariant under the change of variables, Eq. 
74

(4.6), viz.

$$
\prod_{j \neq k}^{6} \frac{d u_{j k}}{u_{j k}}=\prod_{\frac{6}{a_{j}}} \frac{d a_{j}}{a_{j}} \frac{a}{c_{j}}
$$

It is to be noticed that the reduced matrix element, $K$ depends only on a set of six independent channel variables and the covariance conditions are automatically taken into account.

Next we multiply a factor

$$
1=\int_{0}^{\infty} \frac{d \lambda}{\lambda}\left(1-\frac{m_{1}+\cdots+m_{6}}{\lambda}\right)
$$

to the representation (4.7) and subsequently rescale the variables, $m_{j}$ by

$$
m_{j}^{\prime}=\lambda \xi_{j} \cdot\left(1^{\prime}=1, \cdots, 6\right)
$$

Then we find an expression for $F_{C}$ :

$$
F_{c}\left(x, \cdots, x_{6}\right)=\int_{0}^{\infty} \prod \frac{q}{c_{j}} K\left(c_{1}, \cdots, c_{q}\right) \int_{0}^{\infty} \frac{d \lambda}{\lambda} \lambda^{\tau} \int_{0}^{1} \pi d \xi_{j} \text {. }
$$

$-\xi_{j}^{\tau_{j+3}} \delta\left(1-\Sigma^{6} \xi_{j}\right) \exp \left(-i \lambda^{2} \frac{6}{2} x_{j k}^{2} \xi_{j} \xi_{k} v_{j k}\right)$,

where $\tau \equiv \Sigma\left(\tau_{j}+4\right)$ and $v_{j k}=\nu_{j k}\left(a_{j} a_{k}\right)^{-1}$.

(The quantities $V_{j k}$ defined above are functions of the $c_{j}$ alone.)

Let us now Fourier -transform Eq. (4. 8):

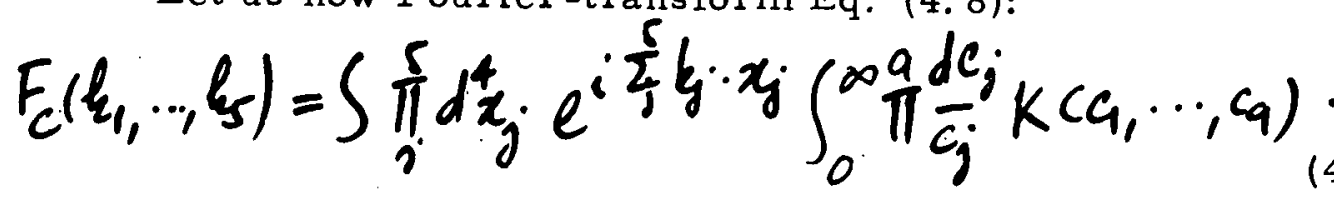

$$
\int_{0}^{\infty} \frac{d \lambda}{\lambda} \lambda^{2} \int_{0}^{1} \frac{6}{\pi} d \xi_{j} \xi_{j} \xi^{+3} \delta\left(1-\underline{\varepsilon} \xi_{j}\right) \exp \left(-i \lambda^{2} \sum_{j i k}^{6} x_{j l}^{2} \xi_{j} \xi_{\xi} v_{j l}\right)_{x_{6}=0} \text {. }
$$

We put $x_{6}=0$ by using translational invariance, thereby one dependent momentum has been eliminated. (Note that we have an overall momentum-energy conservation, $k_{1}+\cdots+k_{6}=0$, 
75

We now define a real and symmetric $5 \times 5$ matrix $Q$ by

$$
X^{t} Q X=\Sigma x_{j k}^{2} \Sigma_{j} \xi_{k} v_{j k},
$$

where $x^{t}=\left(x_{1}, x_{2}, \cdots, x_{5}\right)$ and $x$ is a transpose of the matrix $X$. From the definition it is easily seen that the element ( $j k)$ of $Q$ is given by

$$
Q_{j k}=\delta_{j k} \sum_{l} \xi_{j} v_{j l} \xi_{l}-\xi_{j} v_{j k} \xi_{k} .
$$

As the matrix $Q$ is real and symmetric by definition, there exists an orthogonal matrix which diagonalizes $Q$. Hence the $\boldsymbol{x}$ integrations are easily carried out with the help of the standard

Gaussian integration formula:

$$
\int d^{4} x e^{-i x^{2}}=i \pi^{2} \text {. }
$$

So we get:

$$
\begin{aligned}
& F_{c}\left(k_{1}, \ldots, \xi_{c}\right)=i \pi^{10} \int_{0}^{\infty} \frac{d \lambda}{\lambda} \lambda^{4+\Sigma \tau_{j}} \int_{0}^{\infty} \pi \frac{d c_{j}}{c_{j}} K\left(a_{1}, \cdots, c_{q}\right) . \\
& \cdot \int_{0}^{1} \pi d \xi_{j} \xi_{j}^{\xi_{j}+3} d\left(1-\Sigma \xi_{j}\right)(\operatorname{det} Q)^{-2} \exp \left(\frac{i^{\prime}}{\lambda^{2}} K^{t} Q^{-1} K\right),
\end{aligned}
$$

where $Q^{-t}$ is the inverse matrix of $Q$ and $K^{t}=\left(k_{1}, k_{2}, \ldots, k_{5}\right)$.

Substituting a new variable by $\mu=\left(\lambda^{2}\right)^{-1}$,

$$
\begin{gathered}
F_{c}\left(k_{1}, \cdot \cdots, \xi_{\zeta}\right)=2 i \pi^{10} \int_{0}^{\infty} \frac{d \mu}{\mu} \mu^{-\beta} \int_{0}^{1} \pi d \xi_{j} \xi_{j}^{\tau_{j}+3} \delta\left(1-2 \xi_{j}\right) . \\
\cdot \int_{0}^{\infty} \pi \frac{d \varepsilon_{j}}{c_{j}} k\left(c_{1}, \cdots, c_{q}\right)(\operatorname{der} Q)^{-2} \exp \left(i^{\prime} \mu K^{t} Q^{-1} K\right),
\end{gathered}
$$

where $\beta=2+\frac{1}{2} \tau \tau_{j}$ (or $\frac{1}{2} \tau-10$ ).

Notice that all the momenta are entirely contained in the factor, 
$(K Q K)^{\beta}$. This allows one to obtain a scaling law and a parton-like structure of the inclusive cross sections as we will see in the next section.

Evidently, the dynamics is entirely contained in the generalized reduced matrix element, $\mathrm{K}\left(\mathrm{c}_{1} \ldots \mathrm{c}_{9}\right)$ which cannot be determined by conformal symmetry alone. Without having a dynamical model, we are unable to proceed further with Eq. (4.13). In the following, however, we will study two simple models for the matrix element $\mathrm{K}\left(\mathrm{c}_{1} \ldots \mathrm{c}_{9}\right)$; the skeleton amplitude defined in DK and the independent correlation model. We may hope that at least some qualitative aspects of the results that we find by studying these models remain in a future more complete theory.

\section{3 Some General Properties and the Skeleton Amplitude}

Let us begin with the kinematics of the single particle inclusive reaction

$$
p_{1}+p_{2} \rightarrow q+X
$$

where $X$ symbolize all the rest of the particles that we do not detect. We also denote incident particles (say, scalar nucleons) and an outcoming particle (say, $\pi$ ) by their momenta for the notational simplicity. It is not difficult to see that the inclusive cross section is proportional to the absorptive part of a six point forward scattering amplitude with momenta assigned as follows:

$$
\begin{array}{ll}
k_{1}=p_{1}, & k_{4}=-p_{2}, \\
k_{2}=q, & k_{5}=-q, \\
k_{1}=p_{2}, & k_{6}=-p_{1} .
\end{array}
$$

On choosing the third component of $p_{1}$ different from zero in the center 
of the mass system, the following asymptotic relations are easily shown to hold at high energies:

$$
\begin{aligned}
& p_{1} \simeq\left(p_{1} 0,0, p\right), \\
& p_{2}=(p, 0,0,-p), \\
& q \simeq\left(q_{1}, 8_{T}, q_{0} \cos \theta\right) ;\left|q_{T}\right|=8 \mathrm{cin} \theta .
\end{aligned}
$$

The kinematical invariants, $s, t$, and $u$ are defined as usual:

$$
\begin{aligned}
& s=\left(r_{1}+p_{2}\right)^{2} \simeq 4 p^{2}, \\
& t=\left(p_{1}-8\right)^{2} \simeq-4 p 8 \sin ^{2} \frac{\theta}{2}, \\
& u=\left(p_{2}-8\right)^{2} \simeq-4 p q \cos ^{2} \frac{\theta}{2} .
\end{aligned}
$$

We also define scale variables, $x$ :

$$
x=-\frac{t}{s}, y=-\frac{u}{s}
$$

Note the following asymptotic relations:

$$
x+y=\frac{28}{\sqrt{5}}, \quad x y=\frac{8_{T}^{2}}{5} \text {. }
$$

Thus we see that $x+y \rightarrow 1$ at the boundary of the phase space. At $\theta=90^{\circ}$ we have $x=y$.

Turning our attention to the conformal amplitude (4.13) again, we notice that the six-point function entering the expression of the inclusive cross section can be written in the form with the above defined kinematics.

$$
F_{c}\left(p_{1}, 8, p_{2},-p_{2},-8\right)=s^{\beta} \Phi\left(x, y ; \frac{m_{j}^{2}}{s}\right) .
$$

Taking the absorptive part of the amplitude (4.19) in s, we obtain a general scaling law for the inclusive cross section, 


$$
q^{0} \frac{d^{3}}{d^{3} q}=s^{-1+\beta} F(x, y)
$$

provided we are allowed to let the external masses, mj go to zero. Whether this step is permissible or not depends on the function $\mathrm{K}\left(\mathrm{c}_{1} \ldots \mathrm{c}_{9}\right)$. For the moment we assume that $\mathrm{Eq} .(4.19)$ is free of such infrared divergences. (However, as we will see shortly, this is not the case for the skeleton amplitude.) So we have, in some sense, demonstrated that the scaling law is a consequence of a rather general principle like an approximate conformal invariance of the theory. In order to get more specific information about the scaling function $F(x, y)$, we need a model for $\mathrm{K}\left(\mathrm{c}_{1} \ldots \mathrm{c}_{9}\right)$.

Before doing this, one more interesting consequence of the conformal invariance of the theory is to be mentioned. Namely, we notice that Eq. (4.13) may be rewritten in the form:

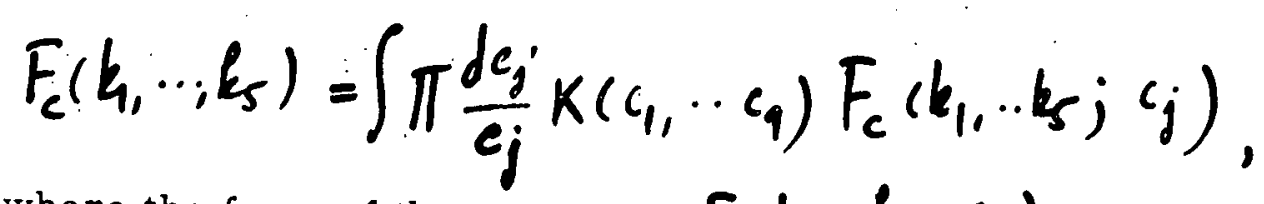

where the form of the amplitude $F_{c}\left(k_{1} \cdot . k_{5} ; c_{j}\right)$ can be read off by comparing Eq. (4.21) with Eq. (4.13). It is important to realize that the function $F_{c}\left(k_{1} \cdot \cdot k_{s} ; c_{j}\right)$ itself is a plysically meaningful $(\operatorname{SU}(2,2)$ invariant) amplitude for any set of the real numbers, $\boldsymbol{c}_{\mathbf{1}}, \ldots, \boldsymbol{c}_{\mathbf{q}}$. Equivalently, the function $\mathrm{K}$ is independent of all the kinematic variables. (From the geometricall point of view, this is a consequence of the fact that conformal transformations act transitively on Minkowski space. If we had required Poincaré invariance of the amplitude only, the reduced matrix elements would be functions of the invariant channel energies, 
thus invalidating the whole argument.). Therefore, the discontinuity of $F_{c}$ with respect to the inciclent energy and hence the inclusive cross section may be written as follows:

$$
q^{0} \frac{d^{3} \sigma}{d^{3} q}=q^{\circ} \int \frac{d^{3} \sigma\left(c_{j}\right)}{d^{3} q} d w\left(c_{j}\right)
$$

Thus, we may look at the measured inclusive cross section as an incoherent superposition of "elementary" cross sections, $q^{0} \frac{d \gamma\left(c_{j}\right)}{d \sqrt{d}}$ where the differential probability distribution of the channel correlation parameters, $c_{j}$ is given by

$$
d w\left(c_{j}\right)=\pi \frac{d c_{j}^{\prime}}{c_{j}} K\left(c_{1}, \ldots, c_{q}\right)
$$

(Strictly speaking, for this interpretation to be valid, one should prove that the elementary cross sections and the distribution $d W$ are positive. At this moment, we do not know of the existence of such a proof; how ever, this question of interpretation has little effect on what follows.)

Eq. (4.22) shows us that the inclusive cross sections possess a structure strongly reminiscent of parton models. This structure is obtained without explicitly assuming that partons are "particlelike constituents" of hadrons. In fact, our partons may be identified with the elementary two-point correlation functions which are building blocks of the conformal amplitudes.

So far we have endeavored to obtain general consequences of the SU $(2,2)$ invariant amplitude Eq. (4.13) without being involved in a specific model. We now study simple models for the function $K\left(c_{j}\right)$ to be more practical. As a first try, we may say that the function 
$G\left(\tau_{2}, \cdots, \tau_{56}\right)$ is very smooth and slowly varying in $\tau_{j}$ so that it is approximated by a constant (say, $G=1$ without loss of generality) as a zeroth order. It is easily seen that this implies

$$
G\left(u_{12}, \cdots, u_{56}\right)=\prod^{6} \delta\left(u_{j k}-1\right) .
$$

This, in turn, implies

$$
K^{S}\left(c_{1}, \ldots, c_{q}\right)=\prod^{q} \delta\left(c_{j}-1\right)
$$

as can be se $\in$ n from the definition Eq. (4.7) by some straight-forward calculations.

Therefore, the skeleton amplitude is given by

$$
\begin{aligned}
& F_{c}^{S}\left(\xi_{1}, \cdots, k\right)=2 i \pi^{10} i-\beta P(-\beta) \int_{0}^{1} \pi d \xi_{j} \cdot \xi_{j}{ }^{\tau+3} \text {. } \\
& \text { - } \delta\left(1-\Sigma_{\xi_{j}}\right)\left(\operatorname{det} Q^{s}\right)^{-2}\left(K^{t} Q^{s^{-1}} K\right)^{\beta}
\end{aligned}
$$

where we define the matrix $Q^{S}$ by

$$
Q^{s}=\left.Q\right|_{c_{j}=1} \text {, i.e. } Q_{j l}^{s}=\delta_{j l} \xi_{j}-\xi_{j} \xi_{l} \text {. }
$$

We have also carried out $\mu$ integration in obtaining Eq. (4.26). For tunately enough, it turns out that the $5 \times 5$ matrix, $Q^{s}$ can be inverted by inspection:

$$
\left(Q^{s}\right)_{j k}^{-1}=\delta_{j k} \frac{1}{\xi_{j}}+\frac{1}{\xi_{6}} .
$$

(As a technical point, it is worth mentioning at this point that even though the matrix $Q$ can be inverted in principle by a finite number of algebraic operations, this procedure becomes quite laborious for matrices of the order of five or higher. Therefore, in practice we are forced to make an approximation. We will encounter this situation 
in the next section.).

We also find the determinant of $Q^{s}$ :

$$
\operatorname{det} Q^{3}=\xi_{1} \cdot \xi_{2} \cdots \xi_{6} .
$$

Putting Eqs. (4.26), (4.27) and (4.28) all together, we get the following expression for the skeleton amplitude $\mathrm{F}_{\mathrm{C}}^{\mathrm{S}}$ after some straight-forward manipulations:

$$
\begin{aligned}
F_{c}^{s}\left(k_{1}, \cdots, k_{s}\right) & =i^{1-\beta} \pi^{10} P(-\beta) \int_{0}^{1} \pi d \xi_{j} \xi_{j} \tau_{j}^{+2} \delta\left(1-2 \xi_{j}\right) . \\
& \left(\frac{k^{2}}{\xi_{1}}+\cdots+\frac{k_{6}^{2}}{\xi_{6}}\right)^{\beta} .
\end{aligned}
$$

Thus we learn that the skeleton amplitude is a constant, depending on the external masses, $m_{j}$, only. As a particular case, the forward scattering amplitude which is relevant to the inclusive cross section has no discontinuity in the invariant energy. So the inclusive cross section gets no contribution from the skeleton amplitude. We also see from Eq. (4.29) that the "infrared limit" $\left(k_{j}^{2} \rightarrow 0\right)$ is finite or divergent depending on the sign of $\beta$. It is reasonable to say that the scale dimensions differ little from their canonical values in view of the arguments we presented in the previous chapters. In that case $\beta=2+\frac{\tau_{\tau_{i}}}{2}$ is approximately a round -1 . (Note that $\beta=-1$ if all the dimensions were canonical.) Thus the skeleton amplitude Eq. (4.29) is in fact infrared divergent.

\section{4. Independent Correlation Model}

As we have previously stated, the model, which we describe in this section to obtain more specific information about the scaling function $F(x, y)$ is our version of understanding of the hypothesis about 
82

the "randomness" of the dynamics. The reduced matrix element $K\left(c_{q}, \cdots, c_{q}\right)$ factorizes in this model:

$$
K\left(c_{1}, \cdots, c_{q}\right)=\prod^{q} K_{j}\left(c_{j}\right),
$$

where the functions $K_{j}$ depend on a single variable only.

Incorporating certain symmetry properties which follow from crossing relations and the statistics of the external particles to the present scalar model, we demand that all the meson-nucleon and nucleon-nucleon distributions be equal to each other, respectively. Hence we have:

$$
\begin{aligned}
& K_{1}=K_{2}=K_{4}=K_{5} \equiv U, \\
& K_{3}=K_{6}=K_{1}=K_{4} \equiv v .
\end{aligned}
$$

(Note that $\mathrm{K}_{8}$ is a meson-meson distribution.)

Assuming the validity of the interpretation of $\mathrm{dW}$ as a differential probability distribution, we obtain the following conditions.

$$
0<\int_{0}^{\infty} \frac{d c}{c} U(c), \int_{0}^{\infty} \frac{d c}{c} V(c)<\infty
$$

Eq. (4. 32) implies that we may write in general

$$
U(c)=c^{A} g(c), V(c)=c^{B} h(c) \text {, }
$$

near $c=0$ with positive powers $A$ and $B$, where $g(0), h(0)$ may be finite. This is always possible by choosing A and B appropriately.

Let us now derive a useful approximation to Eq. (4.13) which is essentially a first order perturbation formula around the skeleton. (Remember that the function $\mathrm{K}$ had a form $\mathbb{S}\left(C_{1}-1\right)$ in the 
83

calculations of the skeleton amplitude.) We start by introducing new variables, $\omega_{j k}$ as follows:

$$
\omega_{j k}=v_{j k}-1 \quad\left(1 \leq j^{\prime} k \leq 6\right)
$$

The variables $\boldsymbol{\omega}_{j k}$ are small parameters to make a power series expassion. It is also understood that $\boldsymbol{\omega}_{\mathrm{jk}}$ are to be expressed in terms of the quantities $C_{j}-1$, eng. to first order we have, $\omega_{j j+1}=c_{j}-1, \omega_{j j+2}=c_{j}+c_{j+1}^{\prime}-2, \quad \omega_{j j+3}^{\prime}=c_{6+j}-1$.

The matrix $Q$ can be separated into two pieces by substituting $j k$ by Eq. (4.34):

$$
Q=Q^{s}+R \text {, }
$$

where $Q^{S}$ is the skeleton matrix given previously and

$$
R=Q / v_{j k \rightarrow} \rightarrow \omega_{j k} \text {. }
$$

Now $Q^{-1}$ can be found by a series expansion:

$$
Q^{-1}=\left(Q^{s}\right)^{-1}-\left(Q^{s}\right)^{-1} R\left(Q^{s}\right)^{-1}+\cdots
$$

Since we have already found $\left(Q^{8}\right)^{-1}$ in the previous section, Eq. (4.36) gives $Q^{-1}$ up to first order in $\omega_{j k}$ after some straightforward calculations:

$$
\begin{aligned}
Q_{j k}^{-1} \cong & \cong \frac{1}{\xi_{j}} \delta_{j k}\left(1+\sum_{\ell} \omega_{j l} \xi_{l}\right)+\frac{1}{\xi_{6}}\left(1+\sum_{l} \omega_{6 l} \xi_{l}\right) \\
& +\omega_{6 j}+\omega_{6 k}-\omega_{j l}
\end{aligned}
$$

In what follows, we also put

$$
\operatorname{det} Q=\operatorname{det}\left(Q^{s}+R\right) \simeq \operatorname{det} Q^{s} \text {, }
$$

whereas we retain terms of $O\left(w_{j k}\right)$ in the -rapidly varying -exponential 
function.

From Eqs. (4.13), (4.31) and (4.38) we obtain an approximate expression for the amplitude $F_{c}\left(k_{1}, \cdots, k_{5}\right)$ :

$$
\begin{aligned}
& F_{c}\left(\ell_{1}, \cdots, \xi_{5}\right) \simeq 2 i \pi^{10} \int_{0}^{\infty} \frac{d \mu}{\mu} \mu^{-\beta} \int_{0}^{1} \pi d \xi_{j} \xi_{j}^{\tau j+2} \delta\left(1-\Sigma \xi_{j}\right) \text {. }
\end{aligned}
$$

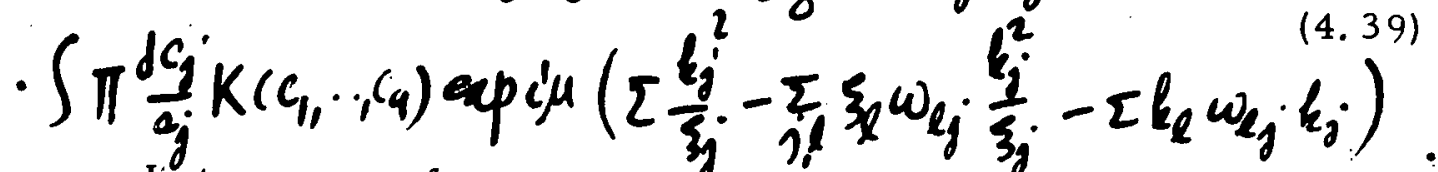

Let us pause for a moment to discuss the validity of the approx-

imate formula Eq. (4.39). Were all the momenta large and nonexceptional (i.e. no finite sum of the external momenta would vanish), we would expert Eq. (4.39) to give a good approximation to the physical (SU $(2,2)$ invariant) amplitude. This is due to the fact that in the region just mentioned, all scalar products of the momenta $k_{i} \cdot k_{j}$ are very large. Hence, the exponential in Eq. (4.39) oscillates very rapidly unless $\boldsymbol{\omega}_{\mathrm{jk}}<1$. (This justifies the assertion that $\boldsymbol{\omega}_{\mathrm{jk}}$ are small parameters.) Unfortunately, however, the region just described is also highly unphysical. (The "deep Euclidean region".) In the physically relevant Minkowskian region of momenta, with $k_{j}^{2}$ fixed, we have no reliable way of estimating the accuracy of our approximation. However, by putting $\mathrm{k}_{\mathrm{j}}^{2}=0$, and working out the respective formulae, we arrive at an approximate amplitude with no evidently unphysical properties. Thus we believe that the approximation considered here gives at least a reasonable qualitative picture of the behaviour of the amplitude.

We immediately notice that the approximation method just derived preserves the scaling property in every order. By consistently neglecting mass terms, the exponent of Eq. (4.39) may be simplified drastically. (For instance, $\xi_{j}$ dependence drops out completely.) Hence we obtain: 
85

$$
\begin{aligned}
\text { Exponent } \rightarrow \sum w_{j l} k_{j} k_{l} & =s\left(c_{1}+c_{2}+c_{4}-c_{5}-c_{1}-c_{q}\right) \\
& -2 t\left(1-c_{6}\right)-2 u\left(1-c_{3}\right) \\
& \equiv s L\left(x, y ; c_{j}\right)
\end{aligned}
$$

To obtain Eq. (4.40) we have used the first order expression of the variables $j \mathrm{jk}$ in terms of $c_{j}-1$. Now the scaling law follows from Eq. (4. 39) just by redefining the variable $\mu$ :

$$
F_{c}\left(p_{1}, q, p_{2},-p_{2},-q\right) \simeq(-s)^{\beta} \psi(x, y),
$$

where $\psi$ is given by:

$$
\begin{aligned}
& \Psi(x, y)=2 i \pi^{10} \int_{0}^{\infty} \frac{d \mu}{\mu} \mu^{-\beta} \int_{0}^{1} d \xi_{j} \cdot \xi_{j} \tau_{j}+2 \delta\left(1-\Sigma \xi_{j}\right) . \\
& \left(L\left(x, y ; c_{j}\right) \text { is } \pi \frac{d c_{j}}{c_{i}} K\left(c_{1}, \cdots, c_{a}\right) \exp i \mu L\left(x, y ; c_{j}\right) .\right.
\end{aligned}
$$

The $\xi_{j}$ integral is easily carried out to give a poly -beta function $B\left(\tau_{1}+2, \cdots \tau_{6}+2\right)$,

$$
B\left(\tau_{1}+2, \cdots, \tau_{t}+2\right)=\frac{\pi P\left(\tau_{j}^{\prime}+2\right)}{P\left(\Sigma\left(2+\tau_{j}\right)\right)} .
$$

Next, the independent correlation model (4.30) tells us that the function $\psi$ can be written in a form.

$$
\begin{aligned}
\psi(x, y)= & 2 i \pi^{10} G B\left(\tau+2, \ldots, \bar{c}_{b}+2\right) \int_{0}^{\infty} \frac{d \mu}{\mu} \mu^{-\beta} . \\
& \cdot \int \pi_{j \neq j} K_{j}\left(c_{j}\right) \frac{d c_{j}}{c_{j}} \exp i \mu L\left(x, y ; c_{j}\right),
\end{aligned}
$$

where, on noticing the absence of $c_{8}$ in the exponent $L$, a parameter $G$ has been introduced by the integral,

$$
G=\int_{0}^{\infty} \frac{d c_{s}}{c_{8}} K_{f}\left(c_{g}\right) \text {. }
$$

Furthermore, let us change variables as follows: 
86

$$
\begin{aligned}
& 2 \mu(1-x-y)=\lambda \\
& \frac{y}{1-x-y} c_{3}=u_{3}, \frac{x}{1-x-y} c_{6}=v_{6}, \frac{1}{2(1-x-y)} c_{6}=u_{k}(4.44) \\
& (k \neq 3,6) .
\end{aligned}
$$

We now obtain the following form for $\psi$ from $E q$. (4. 43) using Eq. (4. 31), (4.33) and (4. 44):

$$
\begin{aligned}
\psi(x, y)= & B(\xi+2, \ldots, \tau+2) p(-\beta)(4 x y)^{-B}[2(1-x-y)]^{\beta+4(A+B)} \\
& \cdot H(x, y),
\end{aligned}
$$

where $\mathrm{H}(x, y)$ is given by

$$
\begin{aligned}
& H(x, y)=G \int \pi \frac{d u_{0}^{\prime}}{u_{j}}\left[u_{1}^{A} g\left(2 u_{1}(1-x-y)\right) \cdots u_{3}^{B} h\left(\frac{u_{3}(1-x-y)}{y}\right) .\right. \\
& \left.\cdots u_{q}^{A} g\left(2 u_{q}(1-\lambda-y)\right)\right]\left(u_{1}+u_{2}+\cdots-u_{q}-1\right)^{\beta} \text {. }
\end{aligned}
$$
further assumptions about the distribution of correlations. However, if $g(0)$ and $h(0)$ are indeed finite, the behaviour of the distribution near the boundary of the phase space $(x+y \rightarrow 1)$ should be dominated by the power $(1-x-y) \beta+4(A+B)$. Similarly, if the functions g and $h$ are reasonably smooth over a substantial range of their arguments, one expects the factor $(x y)^{-B}$ to dominate over the region of small $x$ and $y$. Therefore it is not unreasonable to assume that $\mathrm{H}$ is a slowly varying function in $x$ and $y$. In fact, we are able to fit the experimental data quite satisfactorily with the assumption of $\mathrm{H}$ being a constant. (see Fig. 7)

Finally, calculating the discontinuity of the amplitude $F_{c}$ in the invariant energy $s$, we arrive at the following expression of the single particle distribution:

$$
\begin{aligned}
q^{0} \frac{d^{b}}{d^{3} q} & \sim P(1+\beta)^{-1} B\left(\tau_{1}+2, \cdot \tau_{6}+z\right) s^{-1+\beta}(x y)^{-\beta} . \\
& \cdot(1-x-y)^{\beta+4(A+\beta)} H(x, y),
\end{aligned}
$$


where we have absorbed some constant factors into the function $H$.

It is interesting to notc that the power behaviour, $(x y)^{-B}$, in E.q. (4.46) is of the same type as obtained in the parton model calculations. Our formula Eq. (4.46) also contains a factor, $(1-x-y)^{\beta+4(A+B)}$ which suppress the cross section at the boundary of the phase space $(x+y \rightarrow 1)$ when $\beta+4(A+B)>0$

On combining the scaling law Eq. (4.46) with the dimensional rule (e.g. $\nu_{K}-\nu_{\pi} \sim \frac{1}{M^{2}}\left(M_{K}^{2}-M_{\pi}^{2}\right)$, typically $M \sim \mid$ GeV $)$ obtained in the previous chapter, we find a rather surprising prediction on the inclusive distributions of mesons $(\pi, K\}$,$) at fixed x$ and $y$ :

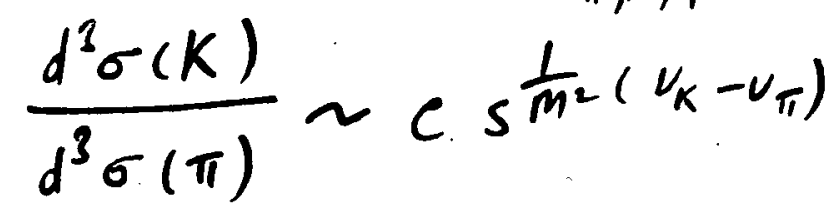

where $\mathbf{C}$, in general, depends on $\mathbf{x}$ and $y$. Similarly for $\boldsymbol{\eta}$. However, under the ad hoc assumption that the constants A, B and the function $H$ are approximately independent of the nature of the particle produced, we obtain the following expression for $C$ by using the fact that for spinless particles:

$$
c=\frac{B\left(1,1-\alpha_{k}, \ldots\right) P\left(-2 \alpha_{\pi}\right)}{B\left(1,1-\alpha_{\pi}, \ldots\right) P\left(-2 \alpha_{k}\right)}
$$

where $\alpha_{j}=1+V_{j}$. We have assumed that the nucleons have a canonical dimension i. e. $V_{N}=-1$.

In order to test our result, in Fig. 7, we have plotted recent CERN-ISR data on $\mathrm{pp} \rightarrow \pi^{\circ}$ anything in three different incident energies, $\sqrt{s}=30.6,44.8$ and 52.7 Gev. Clearly, the scaling law Eq. $(4.46)$ is in good agreement with the data. Our fit is obtained by assuming 


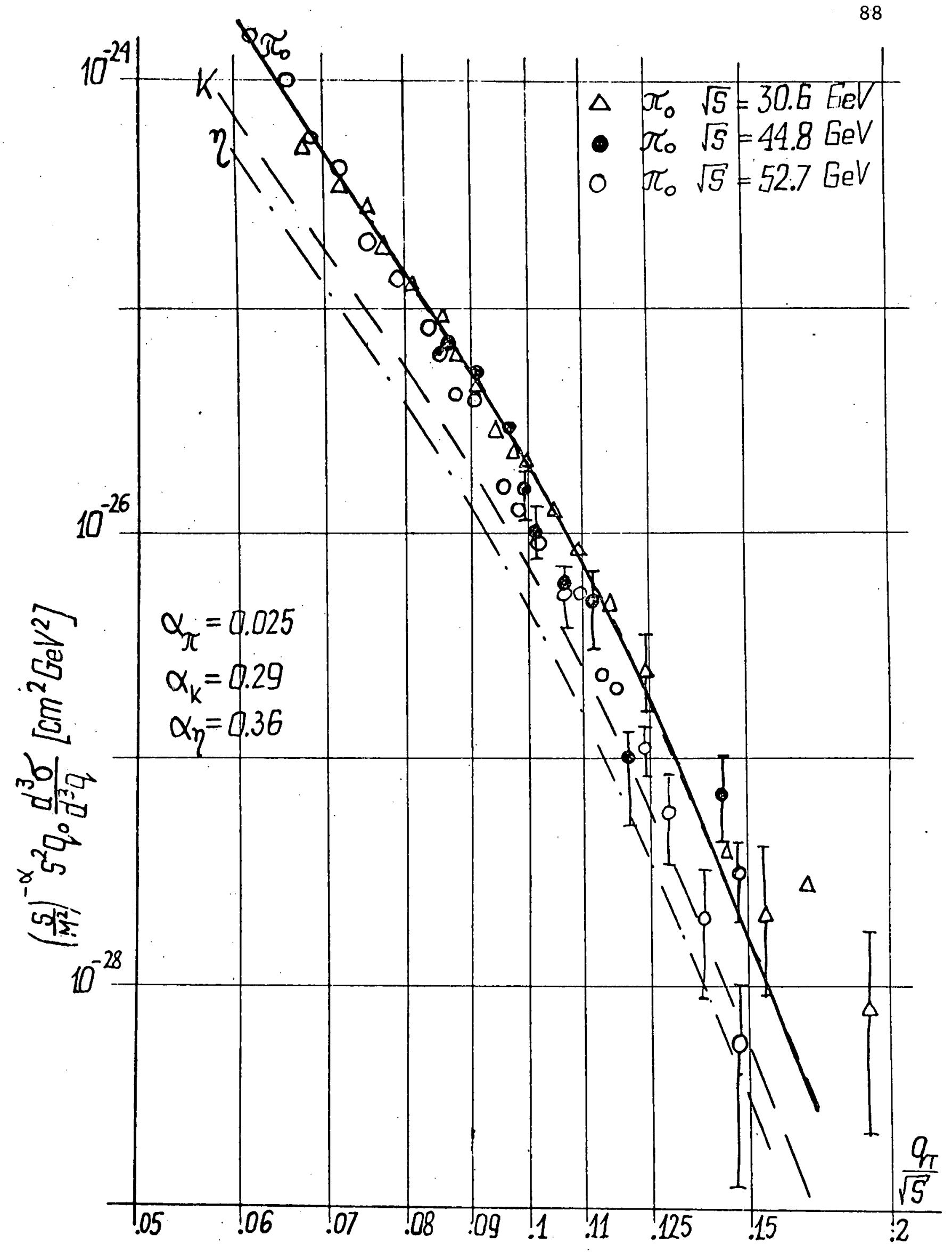

Fig. 7 
$\mathrm{H}(\mathrm{x} y)=$ const and $\mathrm{A}=\mathrm{B} \approx 2.7$. We have also given our predictions on $\mathrm{K}$ and $\boldsymbol{h}$ productions based on Eqs. (4.47), (4.48) in the same figure. At present there are no reliable data available to check these predictions. (Preliminary data obtained by the CERN-Saclay group at the ISR seem to indicate that a) the rate of "heavy particle" production grows with the transverse momentum and may saturate at a ratio around $\sim 1.5$.

b) Comparison with the 24 Gev data indicates a slow increase of heavy particle production with energy. This would be at least qualitatively consistent with our theoretical prediction. However, the data are preliminary and they have been contested by the British-Scandinavian collaboration. At present, no final conclusion can be drawn concerning the validity of this theoretical prediction.) It should be noted that our Eq. (4.47) suggests that at sufficiently high energies and large momentum transfers, one should see an increasing number of low spin, heavy particles produced in any reaction. While this prediction is somewhat surprising in view of the overwhelming dominance of pions at low $\mathrm{q}_{\mathrm{T}}$ as we mentioned in Sec. 4. 1, it does not seem to contradict any known physical principle or experimental result. 


\section{Chapter 5}

Discussion

In the last two chapters we have studied conformal symmetry with an emphasis on its broken character and its physical predictions. Due to the lack of knowledge of the fundamental dynamics of hadrons, we were forced to take a purely phenomenological approach to the problem. Thereby we have developed an effective action theory of studying broken conformal symmetry through a spontaneously broken symmetry mechanism. Assigning hadrons to infinite dimensional representations of $\mathrm{SU}(2,2)$, we were able to recover some essential features of the hadron spectrum. Moreover we predict the ratio $\Gamma_{\text {tot }} / M$ to be a universal constant which seems to agree with present experimental data at least in a qualitative sense. Throughout the calculations, only the qualitative aspects have been emphasized. This is partly because we are not able to provide exact quantitative results within the framework of the present model, and partly because in our present state of knowledge about strong interactions any quantitative estimates are necessarily bound to change before not too long. The main justification of our approximations (based on physical intuition rather than on a logically consistent procedure) would be the consistency of the emerging physical picture with experimental facts. In 
fact, the physical picture seems to be quite attractive. Should the qualitative aspects of the hadron spectrum be correctly described by present theory, we would conclude that it is not physically meaningful to inquire about properties of individual hadron levels in the high mass region. This is because the widths of massive hadron states are predicted to be comparable with their rest masses in such a region. Therefore we should rather concentrate on the investigation of the properties of an "average excited hadron. "This is a familiar concept in nuclear physics, however its accommodation may require substantial changes in the methods and outlook of present day hadron spectroscopy. Broken conformal symmetry also appears to have a considerable predictive power in high entrgy, large momentum transfer hadronic processes. In particular we have considered inclusive reactions in this framework. The predicted scaling law seems to be in reasonable agreement with the data. A far reaching consequence has been obtained from dimensional rules (dilatational trajectories) and the scaling law, predicting more frequent occurence of heavy particles (as opposed to pions) at large transverse momenta. Should this be borne out at least qualitatively by future experiments, it would lend substantial support to our scheme. On the other hand, a deeper understanding of such phenomena would be quite welcome. Recalling that the dimensional rule appears to be quite a new concept in this field and particularly, its appearance in connection with duality, such a theoretical investigation may lead us to a 
completely new domain of strong interactions. Clearly our approach - purely phenomenological in its spirit - is far from being a consistent theory. Yet, the present considerations lead one to conjecture that fine details of the dynamics of complicated relativistic systems - as hadrons appear to be - may be relatively unimportant as far as practically observable properties are concerned. One may recall in this respect that some non-relativistic many-body systems (e.g. an infinite ferromagnet near the Curie-temperature) do occasionally exhibit such a surprising insensitivity to the details of the dynamics. While there are certain formal analogies between a non-relativistic system near the critical point and a conformally invariant relativistic theory, the physical meaning (if there is any) of such analogies is far from being clear at present. Should it happen that the dynamics of hadrons somehow "conspires" to disguise the true nature of the "fundamental" constituents of hadrons (if there are any), the present phenomenological approach may be the only effective starting point towards a dynamical theory of hadrons - perhaps until a radically different type of experimental data will be available. 


\section{Footnotes and References}

$\ddagger$

Research supported in part by the U. S. Atomic Energy Commission under Contract No. AT (11-1) 3285.

The contents of this report are based on a series of papers by G. Domokos, S. Kövesi-Domokos, and B. C. Yunn, JHU preprints COO-3285-6; COO-3285-7. The collaboration of Professor G. Domokos and Dr. S. Kövesi-Domokos is gratefully acknowledged.

1) E. Cunningham, Proc. Lond. Math. Soc. 8, 77 (1909);

H. Bateman, loc. cit. 8, 223 (1910).

2) P. A. M. Dirac, Ann. Math. 37, 429 )1936).

3) L. Page, Phys. Rev. 49, 254, 946 (1936).

4) J. Wess, Nuovo Cimento 18, 1086 (1960); H. A. Kastrup, Ann. Phys. $\underline{7} 388$ (1962); Nucl. Phys. 58, 561 (1964); Phys. Rev. 142 , 1060 (1966); 143, 1041 (1966); 1501189 (1966); G. Mack, Nucl. Phys. B5, 499 (1968); Phys. Lett. , 26B, 575 (1968).

5) G. Mack, loc. cit., see also the review paper by G. Mack and A. Salam, Ann. Phys. 53, 174 (1969).

6) J. D. Bjorken, Phys. Rev. 179, 1547 (1969).

7) E. D. Bloom et al, Phys. Rev. Lett. 23, 950 (1969); M. Breidenbach, MIT, thesis, 1970 (unpublished).

8) K. G. Wilson, Phys. Rev. 179, 1499 (1969).

9) R. Brandt and G. Preparata, Nuel. Phys., B27, 541 (1971);

Y. Frishman, Phys. Rev. Lett. 25, 966 (1971). 
References - Cont'd.

10) S. Ferrara, R. Gatto and A. F. Grillo, Phys. Lett. $\underline{36 B}, 124$ (1971); S. Ferrara, A. F. Grillo, G. Parisi and R. Gatto, Phys. Lett. 38B, 333 (1972).

11) K. G. Wilson, Phys. Rev. D2, 1471, 1478 (1970).

12) R. Jackiw, Phys. Rev. D3, 2005 (1971).

13) See e.g. K. G. Wilson, Cornell preprint, CLNS-198 (1972);

P. K. Mitter, University of Maryland preprint (1973).

14) A. A. Migdal, Phys. Lett. 37B, 386 (1971).

15) G. Mack and I. T. Todorov, Trieste preprint, IC/71/139, (1971); G. Mack and K. Symanzik, DESY preprint, DESY 72/20 (1972); K. Symanzik, Lett. Nuovo Cimento $\underline{3}$, 734 (1972).

16) G. Domokos and S. Kövesi-Domokos, JHU preprint COO-3285-2, (1972) (to be published); hereafter we refer to this reference as DK.

17) J. Goldstone, Nuovo Cimento, 19, 154 (1961).

18) P. G. O. Freund and Y. Nambu, Phys. Rev. 174, 1741 (1968).

19) B. Zumino, in "Lectures on Elementary Particles and Quantum Field Theory", Vol. 2, ed. by S. Deser, M. Grisaru and H. Pendleton, MIT Press, Cambridge (1970).

20) F. Guirsey, Ann. Phys. 24, 211 (1963).

21) C. G. Callan, S. Coleman and R. Jackiw, Ann. Phys. 5942 (1970). 
References - Cont'd.

22) In this limit the field $\varphi$ remains massive. This can be seen by noticing $m_{\varphi}^{2}=\lambda_{0} \lambda_{2}$.

23) E. Del Giudice, P. Di Vecchia, S. Fubini and R. Musto, Nuovo Cimento 12A, 813 (1972).

24) L. Brillouin, Joum. de Phys. $\underline{4}, 1$ (1963); E. P. Wigner, Math. u. Naturw. Anz. der Ung. Akad. der Wiss, 53, 477 (1935).

25) See e.g. P. Carruthers, Phys. Reports $1 \mathrm{C}, 1$ (1971).

26) E. P. Wigner, in "Group Theoretical Concepts and Methods in Elementary Particle Physics," ed. by F. Gürsey, pp. 37, Gordon and Breach, New York, (1962).

27) G. Mack and A. Salam, ref. 5.

28) G. Domokos, S. Kövesi-Domokos and F. Mansouri, Phys. Rev. D1, 500, 512 (1970).

29) This is the principal series $d_{2}$ according to the classification of Graev. Cf. M. L. Graev, Dokl. Akad. Nauk SSSR, 98, 517 (1954); A. Esteve and P. G. Sona, Nuovo Cimento 32, 473 (1964).

30) In the framework of a canonical field theory such an assignment is required for the massless free field theory to be conformally invariant.

31) Even though $\lambda \phi^{4}$-theory is not scale invariant in the perturbation theory, we can still talk about anomalous dimensions to lowest order in $\lambda$. 
References - Cont'd.

32) G. Jona-Lasinio, Nuovo Cimento 34, 1790 (1964); B. Zumino, ref. 19 .

33) K. Nishijima, Phys. Rev. 111, 995 (1958).

34) I. Montvay, Phys. Rev. D3, 2532 (1971).

35) K. Symanzik, ref. 15.

36) S. Ferrara, R. Gatto and A. F. Grillo, Frascati preprint, LNF-71/79 (1971).

37) This is essentially the Wigner-Eckart theorem for $\mathrm{SU}(2,2)$.

See e.g. E. Schreier, Phys. Rev. D3, 980 (1971).

38) R. J. Eden et al., The Analytic S-Matrix, Cambridge at the Uni versity Press, (1966).

39) F. Mansouri, Nuovo Cimento, 3A, 220 (1971).

40) See e.g. K. Symanzik, Commun. Math. Phys. 18, 227 (1970); 23, 49 (1971).

41) I. M. Gelfand and G. E. Shilov, Generalized Functions, Vol. 1, Ch. III, Academic Press, New York and London (1964).

42) I. T. Todorov and R. P. Zaikov, Journ. Math. Phys. 10, 2014, (1969).

43) A. Erdélyi; W. Magnus, F. Oberhettinger and F. G. Tricomi, Higher Transcendental Functions, Vol. 1, McGraw-Hill, New York (1953). 
References - Cont'd.

44) A. Erdélyi, W. Magnus, F. Oberhettinger and F. G. Tricomi, Higher Transcendental Functions, Vol. 2, Ch. X, McGrawHill, New York (1953).

45) A. Erdélyi, W. Magnus, F. Oberhettinger and F. G. Tricomi, Table of Integral Transforms, Vol. 2, Ch. XVI, McGrawHill, New York (1954).

46) E. C. Titchmarsh, Theory of Functions, Ch. V, Oxford University Press, (sec. ed.) (1958).

47) A. R. Forsyth, Functions of Two Variables, Ch. III, Cambridge: at the University Press (1914).

48) P. Söding et al., Review of Particle Properties, Phys. Lett. 39B (1972).

49) For a general review, see e.g. L. Van Hove, Phys. Reports 1C, 347 (1971); D. Horn, Phys. Reports, 4C, 1 (1972).

50) CERN-Columbia-Rockefeller collaboration, contribution to the NAL-Chicago Conference (Sept. 1972) and at the Vanderbilt Conference (Nashville, Tenn.), (1973).

51) R. P. Feynman, Phys. Rev. Lett. 23, 1415 (1969) and in "High Energy Collisions," ed. by C. N. Yang et al., Gordon and Breach, New York (1970).

52) R. P. Feynman, loc. cit.; J. D. Bjorken and E. A. Paschos, Phys. Rev. 185, 1975 (1969). 
References - Cont'd.

53) See e.g. J. F. Gunion, S. J. Brodsky and R. Blankenbecler, Phys. Rev. D6, 2652 (1972); P. V. Landshoff and J. C.

Polkinghorne, Cambridge preprints, DAMTP 72/43, 72/48, (1972); D. Cline, F. Halzen and M. Waldrop, University of Wisconsin preprint (1973).

54) A. H. Mueller, Phys. Rev. D2, 2963 (1970). 